\title{
Stochastic gravito-inertial modes discovered by CoRoT in the hot Be star HD 51452
}

\author{
C. Neiner ${ }^{1}$, M. Floquet ${ }^{2}$, R. Samadi ${ }^{1}$, F. Espinosa Lara $^{3}$, Y. Frémat ${ }^{4}$, S. Mathis ${ }^{5,1}$, B. Leroy ${ }^{1}$, B. de Batz ${ }^{1}$, M. Rainer ${ }^{6}$, \\ E. Poretti ${ }^{6}$, P. Mathias ${ }^{3}$, J. Guarro Fló ${ }^{7}$, C. Buil ${ }^{8}$, J. Ribeiro ${ }^{9}$, E. Alecian ${ }^{1}$, L. Andrade ${ }^{10}$, M. Briquet ${ }^{1,11}$, \\ P. D. Diago ${ }^{12,14}$, M. Emilio ${ }^{13}$, J. Fabregat ${ }^{14}$, J. Gutiérrez-Soto ${ }^{12,15,1}$, A.-M. Hubert ${ }^{2}$, E. Janot-Pacheco ${ }^{10}$, \\ C. Martayan ${ }^{16}$, T. Semaan ${ }^{2}$, J. Suso ${ }^{14}$, and J. Zorec ${ }^{17}$
}

1 LESIA, UMR 8109 du CNRS, Observatoire de Paris, UPMC, Univ. Paris Diderot, 5 place Jules Janssen, 92195 Meudon Cedex, France e-mail: Coralie.Neiner@obspm.fr

2 GEPI, UMR 8111 du CNRS, Observatoire de Paris, Univ. Paris Diderot, 5 place Jules Janssen, 92195 Meudon Cedex, France

3 Université de Toulouse, UPS-OMP, IRAP, CNRS, 14 avenue Edouard Belin, 31400 Toulouse, France

${ }^{4}$ Royal Observatory of Belgium, 3 avenue circulaire, 1180 Brussels, Belgium

5 Laboratoire AIM Paris-Saclay, CEA/DSM-CNRS-Université Paris Diderot, IRFU/SAp, Centre de Saclay, 91191 Gif-sur-Yvette Cedex, France

6 INAF - Osservatorio Astronomico di Brera, via E. Bianchi 46, 23807 Merate (LC), Italy

7 ARAS, Astronomical Ring for Access to Spectroscopy

8 Castanet Tolosan Observatory, 6 place Clemence Isaure, 31320 Castanet Tolosan, France

9 R. Venezuela 29, 3 Esq., 1500-618 Lisboa, Portugal

10 University of Sao Paulo, Brazil

11 Institut d'Astrophysique et de Géophysique, Université de Liège, allée du 6 août 17, Bât. B5c, 4000 Liège, Belgium

12 Valencian International University (VIU), Prolongación C/ José Pradas Gallen s/n, edificio B piso 2, 12006 Castellón de la Plana, Spain

13 Observatório Astronômico/DEGEO, Universidade Estadual de Ponta Grossa, Av. Carlos Cavalcanti, 4748 Ponta Grossa, Paraná, Brazil

14 Observatori Astronòmic de la Universitat de València, Edifici Instituts d’Investigatió, Poligon La Coma, 46980 Paterna, València, Spain

15 Instituto de Astrofísica de Andalucía (CSIC), Glorieta de la Astronomía s/n, 18008 Granada, Spain

16 ESO, Alonso de Cordova 3107, Vitacura, Casilla 19001, Santiago 19, Chile

17 Institut d'Astrophysique de Paris, UMR 7095, CNRS, Université Pierre \& Marie Curie, 98bis Boulevard Arago, 75014 Paris, France

Received 14 June 2012 / Accepted 7 August 2012

\section{ABSTRACT}

Context. Be stars are rapidly rotating stars with a circumstellar decretion disk. They usually undergo pressure and/or gravity pulsation modes excited by the $\kappa$-mechanism, i.e. an effect of the opacity of iron-peak elements in the envelope of the star. In the Milky Way, p-modes are observed in stars that are hotter than or equal to the B3 spectral type, while g-modes are observed at the B2 spectral type and cooler.

Aims. We observed a B0IVe star, HD 51452, with the high-precision, high-cadence photometric CoRoT satellite and high-resolution, ground-based HARPS and SOPHIE spectrographs to study its pulsations in great detail. We also used the lower resolution spectra available in the BeSS database.

Methods. We analyzed the CoRoT and spectroscopic data with several methods: CLEAN-NG, FREQFIND, and a sliding window method. We also analyzed spectral quantities, such as the violet over red (V/R) emission variations, to obtain information about the variation in the circumstellar environment. We calculated a stellar structure model with the ESTER code to test the various interpretation of the results.

Results. We detect 189 frequencies of variations in the CoRoT light curve in the range between 0 and $4.5 \mathrm{c} \mathrm{d}^{-1}$. The main frequencies are also recovered in the spectroscopic data. In particular we find that HD 51452 undergoes gravito-inertial modes that are not in the domain of those excited by the $\kappa$-mechanism. We propose that these are stochastic modes excited in the convective zones and that at least some of them are a multiplet of r-modes (i.e. subinertial modes mainly driven by the Coriolis acceleration). Stochastically excited gravito-inertial modes had never been observed in any star, and theory predicted that their very low amplitudes would be undetectable even with CoRoT. We suggest that the amplitudes are enhanced in HD 51452 because of the very rapid stellar rotation. In addition, we find that the amplitude variations of these modes are related to the occurrence of minor outbursts.

Conclusions. Thanks to CoRoT data, we have detected a new kind of pulsations in HD 51452, which are stochastically excited gravito-inertial modes, probably due to its very rapid rotation. These modes are probably also present in other rapidly rotating hot Be stars.

Key words. stars: emission-line, Be - stars: individual: HD 51452 - stars: oscillations - stars: rotation 


\section{Introduction}

Be stars are late $\mathrm{O}, \mathrm{B}$, or early A stars that host a circumstellar decretion disk fed by discrete mass loss events (called outburts). The ejections and disk produce emission lines in the optical spectrum of Be stars, as well as infrared excess in the spectral energy distribution. We refer the reader to Porter \& Rivinius (2003) for a complete review of Be stars, as well as Neiner et al. (2011b) for the most recent compilation of work on this topic.

Be stars are known to pulsate. As of today, the only driving mechanism of pulsations in Be stars was thought to be the $\kappa$-mechanism, i.e. an effect of the opacity of iron-peak elements in the envelope of the star (Dziembowski \& Pamyatnykh 1993; Dziembowski et al. 1993). Pressure/acoustic (p) and gravity (g) modes can be auto-excited depending on the depth at which the iron opacity bump is situated in the envelope, i.e. depending on the temperature of the star. For p-modes, pressure is the main restoring force, while for g-modes the restoring force is buoyancy. In the Milky Way, p-modes are observed in hot Be stars down to the B3 spectral type, while g-modes are observed in cooler Be stars from the B2 spectral type downwards. Therefore, B2-3 stars can be hybrid pulsators with both p- and g-modes.

Space missions providing high-precision, high-cadence photometric data over long periods of time, such as CoRoT and Kepler, allow us to study pulsations in all classes of stars including Be stars (e.g. Gutiérrez-Soto et al. 2009; Neiner et al. 2009). These missions have already provided important results. In the case of Be stars, a major result was obtained by Huat et al. (2009), who showed that there is a strong correlation between the ejections of material from Be stars and the presence and amplitude variations of pulsations.

In this paper, we describe the observations of the hot Be star HD 51452 obtained with CoRoT and ground-based spectroscopy (Sect. 2). We present the star and its circumstellar variations (Sect. 3), as well as the results of a time-series analysis performed on these data (Sect. 4). We then discuss the results in terms of excitation mechanism of pulsations (Sect. 5). Finally, conclusions are given in Sect. 6.

\section{Observations}

HD 51452 has been observed with the CoRoT satellite during 114.4 consecutive days. Simultaneously, ground-based observations were obtained during about one month in spectroscopy with HARPS (ESO, Chile) and SOPHIE (OHP, France). Additional spectra, available in the BeSS database (Neiner et al. 2011a), have also been collected, with a scarcer sampling but over the whole CoRoT run. A log of the groundbased observations is provided in Table 1 .

\subsection{CoRoT space-based photometry}

CoRoT was launched on December 27, 2006. The raw photometric data acquired with CoRoT have been reduced by the CoRoT team. The corrections applied on the data are the subtraction of the offset and background, the correction from the cross-talk

* The CoRoT space mission, launched on December 27, 2006, has been developed and is operated by CNES, with the contribution of Austria, Belgium, Brazil, ESA (RSSD, and Science Programs), Germany, and Spain. This work uses observations partly made with the HARPS instrument at the 3.6-m ESO telescope (La Silla, Chile) in the framework of the LP182.D-0356, as well as data obtained with Sophie at $\mathrm{OHP}$ and from the BeSS database.

$\star \star$ Table 3 is available in electronic form at

http://www . aanda.org
Table 1. Journal of HARPS, SOPHIE, and BeSS observations of HD 51452 obtained during the CoRoT observing run between October 2008 and March 2009.

\begin{tabular}{lcc|ccc}
\hline \hline Date & $\#$ & Instr & Date & $\#$ & Instr \\
\hline Oct. 5 & 1 & BeSS & Jan. 12 & 5 & Sophie \\
Oct. 24 & 1 & BeSS & Jan. 13 & 4 & Sophie \\
Oct. 26 & 1 & BeSS & Jan. 14 & 3 & Sophie \\
Nov. 15 & 1 & BeSS & Jan. 14 & 1 & BeSS \\
Nov. 29 & 1 & BeSS & Jan. 15 & 5 & Sophie \\
Nov. 30 & 1 & BeSS & Jan. 16 & 4 & Sophie \\
Dec. 8 & 1 & BeSS & Jan. 16 & 1 & BeSS \\
Dec. 12 & 6 & Sophie & Jan. 17 & 2 & Sophie \\
Dec. 14 & 7 & Harps & Jan. 21 & 1 & BeSS \\
Dec. 15 & 7 & Harps & Jan. 27 & 2 & BeSS \\
Dec. 16 & 6 & Harps & Feb. 10 & 1 & BeSS \\
Dec. 17 & 6 & Sophie & Feb. 11 & 1 & BeSS \\
Dec. 17 & 7 & Harps & Feb. 13 & 1 & BeSS \\
Dec. 18 & 5 & Sophie & Feb. 14 & 1 & BeSS \\
Dec. 18 & 7 & Harps & Feb. 17 & 1 & BeSS \\
Dec. 19 & 7 & Harps & Feb. 18 & 1 & BeSS \\
Dec. 20 & 6 & Harps & Feb. 20 & 1 & BeSS \\
Dec. 21 & 7 & Harps & Feb. 21 & 1 & BeSS \\
Dec. 22 & 1 & BeSS & Feb. 23 & 1 & BeSS \\
Dec. 22 & 7 & Harps & Feb. 26 & 1 & BeSS \\
Dec. 23 & 7 & Harps & Feb. 27 & 1 & BeSS \\
Jan. 3 & 7 & Harps & Mar. 13 & 1 & BeSS \\
Jan. 4 & 8 & Harps & Mar. 15 & 1 & BeSS \\
Jan. 5 & 8 & Harps & Mar. 16 & 1 & BeSS \\
Jan. 6 & 8 & Harps & Mar. 18 & 1 & BeSS \\
Jan. 7 & 7 & Harps & Mar. 23 & 1 & BeSS \\
Jan. 10 & 6 & Sophie & Mar. 25 & 1 & BeSS \\
Jan. 11 & 7 & Sophie & & & \\
\hline & & & & & \\
\hline
\end{tabular}

Notes. Column 1 indicates the night of the observation, Col. 2 indicates the number of spectra acquired that night, and Col. 3 shows the instrument used.

effect, the transformation from ADU to electrons, and the corrections of the exposure time variations, jitter effect, and outliers. For more details, see Auvergne et al. (2009) and Samadi et al. (2007).

The CoRoT satellite observed HD 51452 in its LRA2 (Long Run Anticenter \#2) asteroseismology field during 114.4 continuous days from November 13, 2008 to March 8, 2009. The CoRoT light curve is shown in Fig. 1.

In the asteroseismology fields, CoRoT obtains one measurement each second. Averaged measurements every $32 \mathrm{~s}$ are then calculated and constitute the light curve, with time converted to the heliocentric system. A flag is assigned to each data point to indicate if this measurement has been obtained during a proton impact, the South Atlantic Anomaly (SAA) crossing, a transition of the satellite from light to penumbra or vice versa, a large jitter correction, or a light-curve discontinuity due to temperature effects. In this paper, we analyzed the light curve with data points with flag 0 only, i.e., taken in perfect conditions, which corresponds to 278058 photometric measurements.

The light curve shows a linear trend of about 0.1 mmag per day (i.e. 12 mmag over the observing run), visible in the data of other CoRoT targets as well, which are attributed to the effect of the aging of some instrument parts (CCDs and/or optics). This trend was removed before analyzing the data.

\subsection{Ground-based spectroscopy}

Ground-based spectroscopic observations were obtained with HARPS, SOPHIE, and from BeSS. Figure 1 indicates the 


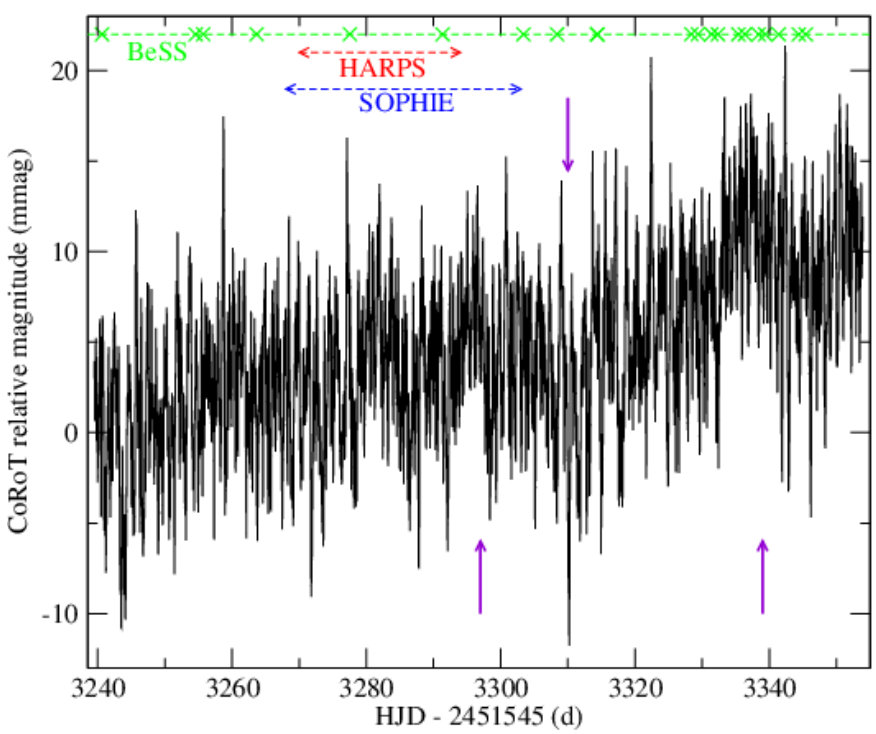

Fig. 1. CoRoT light curve of the hot Be star HD 51452. The periods when HARPS, SOPHIE, and BeSS data were acquired are also indicated with dashed lines. For BeSS, the exact times of acquisition of spectra are marked with crosses. The 3 purple arrows indicate the time of minor outburts.

Table 2. Stellar parameters of HD 51452.

\begin{tabular}{lc}
\hline \hline Parameter & Value \\
\hline$T_{\text {eff }}^{\text {surf }}(\mathrm{K})$ & $29500 \pm 500$ \\
$\log g^{\text {surf }}$ & $3.95 \pm 0.04$ \\
$R\left(R_{\odot}\right)$ & $\sim 7$ \\
$v \sin i\left(\mathrm{~km} \mathrm{~s}^{-1}\right)$ & $315.5 \pm 9.5$ \\
$i\left(^{\circ}\right)$ & $49 \pm 10$ \\
$f_{\text {rot }}\left(\mathrm{c} \mathrm{d}^{-1}\right)$ & $1.22 \pm 0.15$ \\
\hline
\end{tabular}

periods during which these spectra were obtained during the CoRoT observing run.

\subsubsection{HARPS}

HARPS is installed on the 3.6-m telescope of ESO at $\mathrm{La}$ Silla in Chile. We collected 106 spectra of HD 51452 with an exposure time of $1200 \mathrm{~s}$ per spectrum from December 14, 2008 to January 7, 2009. The resolution is $R=80000$, and the fast readout noise was used. The signal-to-noise ratio of the spectra ranges from 92 to 179 .

The spectra were reduced using both the ESO pipeline and a semi-automatic MIDAS pipeline (Rainer 2003). However, we noticed strong fringes in the resulting data. A detailed investigation revealed a misalignement of a filter on the path of the flat-field lamp, which produced fringes in the flat-field data introduced during the reduction process in the stellar spectra. A rereduction of the data with new flatfields after the ESO staff realigned the filter still produced small fringes. Finally, the filter was completely removed by ESO from the optical path of the flat-field lamp, and the final reduction was performed with newly acquired flat-field data. Consequently flatfields used for the reduction were obtained in July 2009, i.e. several months after the stellar observations. However, HARPS is known to have ultra-high internal stability (e.g. Wildi et al. 2011) and the later acquisition of the flatfields does not seem to have affected the resulting spectra.

\subsubsection{SOPHIE}

SOPHIE is a cross-dispersed echelle spectrograph installed on the 1.93-m telescope of OHP (Observatoire de Haute-Provence) in France. We used the high efficiency (HE) mode with a resolution of $R=40000$. Fifty three observations were obtained between December 12, 2008 and January 16, 2009. The exposure time varied from 1800 to $3600 \mathrm{~s}$ depending on the weather conditions during the observations. The resulting signal-to-noise ratio varies from 38 to 276 . The usual bias, flatfield, and wavelength calibration were performed using the OHP pipeline.

\subsubsection{BeSS spectra}

Simultaneously with the CoRoT observations, from October 5, 2008 to March 25, 2009, $30 \mathrm{H} \alpha$ spectra were collected by amateur astronomers with telescopes from 28 to $41 \mathrm{~cm}$ in diameter and a resolution $R=6000$. These reduced data are available in the BeSS database (Neiner et al. 2011a).

\section{The Be star HD 51452}

\subsection{Stellar parameters}

HD 51452 is a magnitude $V=8.08$ classical Be star of spectral type B0IVe. The spectra presented here indeed show very weak He II 4200 and 4542 absorption lines and a bit stronger He II 4686 line, which indicate that the star has an effective temperature somewhat hotter than $27000 \mathrm{~K}$ (Martins 2011). The He II 4686 line can also be influenced by the stellar wind.

In the frame of the preparation to the CoRoT mission, Frémat et al. (2006) determined the apparent stellar parameters of HD 51452 and found that the effective temperature is $T_{\text {eff }}=30000 \pm 1500 \mathrm{~K}$, the surface gravity is $\log g=$ $3.88 \pm 0.15$, and the projected rotational velocity is $v \sin i=$ $298 \pm 20 \mathrm{~km} \mathrm{~s}^{-1}$. By accounting for gravitational darkening effects, the same authors showed that the value of the parent nonrotating counterpart (pnrc) stellar parameters of HD 51452 are $T_{\text {eff }}=$ [30500-31 500] K, $\log g=$ [3.99-4.04], $v \sin i=[306-325] \mathrm{km} \mathrm{s}^{-1}$, and $i=[39-59]^{\circ}$ depending on the adopted value of the $\Omega / \Omega_{\mathrm{c}}$ ratio, which was assumed to vary from 0.8 to 0.99 . In terms of pnrc surface-averaged parameters (i.e. values of interest when considering stellar evolutionary tracks that take fast rotation effects into account), this gave $T_{\mathrm{eff}}^{\text {surf }}=[29000-30000] \mathrm{K}$ and $\log g^{\text {surf }}=[3.91-3.99]$. These parameters correspond to a star with stellar radius $R \sim$ $7 R_{\odot}$ with estimated rotation frequency $f_{\mathrm{rot}}=[1.07-1.37] \mathrm{c} \mathrm{d}^{-1}$. These determinations were carried out using lower spectral resolution and signal-to-noise data, but the analysis of our new high-quality echelle spectra confirms the results by Frémat et al. (2006, see Fig. 2).

However, since our spectroscopic data are of much higher quality (resolution, signal-to-noise, and wavelength coverage) than those used by Frémat et al. (2006), while Frémat et al. (2006) assumed solar metallicity, we were able to test if the metallicity could be significantly higher than solar. We used the procedure described in Frémat et al. (2006) to calculate synthetic spectra. Figure 2 shows the fit of the observed spectrum with solar metallicity and microturbulence of $\xi_{\mathrm{t}}=9 \mathrm{~km} \mathrm{~s}^{-1}$, as well as a fit with the abundance of $\mathrm{C}, \mathrm{N}, \mathrm{O}, \mathrm{Si}, \mathrm{Mg}$, and Fe multiplied by 2 and a microturbulence $\xi_{\mathrm{t}}=5 \mathrm{~km} \mathrm{~s}^{-1}$. We found that the solar metallicity represents the spectrum best: the quadratic deviation for the solar case is 0.0132 , while it is 0.0152 for the enhanced abundances. 

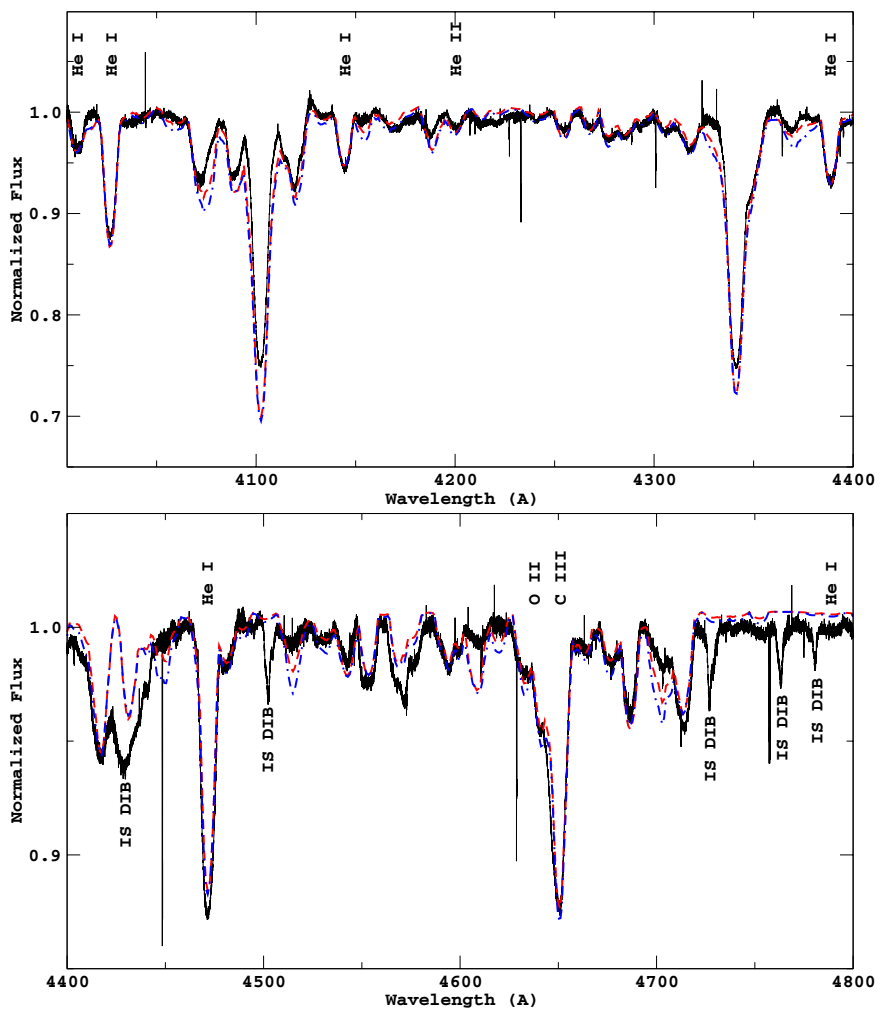

Fig. 2. Fit of the spectrum of HD 51452 using a solar metallicity (red line) and multiplying the abundance of $\mathrm{C}, \mathrm{N}, \mathrm{O}, \mathrm{Si}, \mathrm{Mg}$ and $\mathrm{Fe}$ by 2 (blue line).

\subsection{Circumstellar variations}

Be stars episodically undergo outburts of matter that feed their circumstellar disk. In the CoRoT data of HD 51452, no major outburst was observed, but small outbursts were possibly detected around HJD - $2452545=3297,3310$ and $3339 \mathrm{~d}$ (see Fig. 1). Only the first of these three dates is bracketed well by ground-based data. Spectroscopic HARPS and SOPHIE observations of this period indeed indicated that the $V / R$ ratio underwent a sudden change and the $V+R$ emission underwent a sudden increase (see Fig. 3), confirming that a minor outburst had occurred. The second minor outburst has not been followed with spectroscopy, and for the last minor outburst we only have a few BeSS data points; nevertheless, these few points seem to also show a variation at the outburst time followed by an increase in emission that is clearly visible in the spectra (see Fig. 3).

\section{Pulsation analysis}

\subsection{CoRoT data}

\subsubsection{Fourier analysis of the full light curve}

CoRoT data were analyzed using two Fourier techniques: Clean-NG and FreqFind, both developed within the CoRoT Be team. Both methods were applied to the CoRoT light curve with points with flag 0 only (see Sect. 2). The minimum detectable frequency is $1 / T=0.0087 \mathrm{c} \mathrm{d}^{-1}$ (which is detected as $f_{3}^{\prime}$ ), i.e., $0.1 \mu \mathrm{Hz}$, where $T$ is the length of the CoRoT run. Following Kallinger et al. (2008), the frequency uncertainty of close peaks is $1 /(4 T)=0.0022 \mathrm{c} \mathrm{d}^{-1}$, i.e., $0.025 \mu \mathrm{Hz}$.

Clean-NG uses a stopping criterion based on the relative rate $\mathrm{r}$ of increase in the total power in the clean components from one iteration to the next. We used $r=0.001$ and an oversampling of 4 . For a detailed description of this method, we

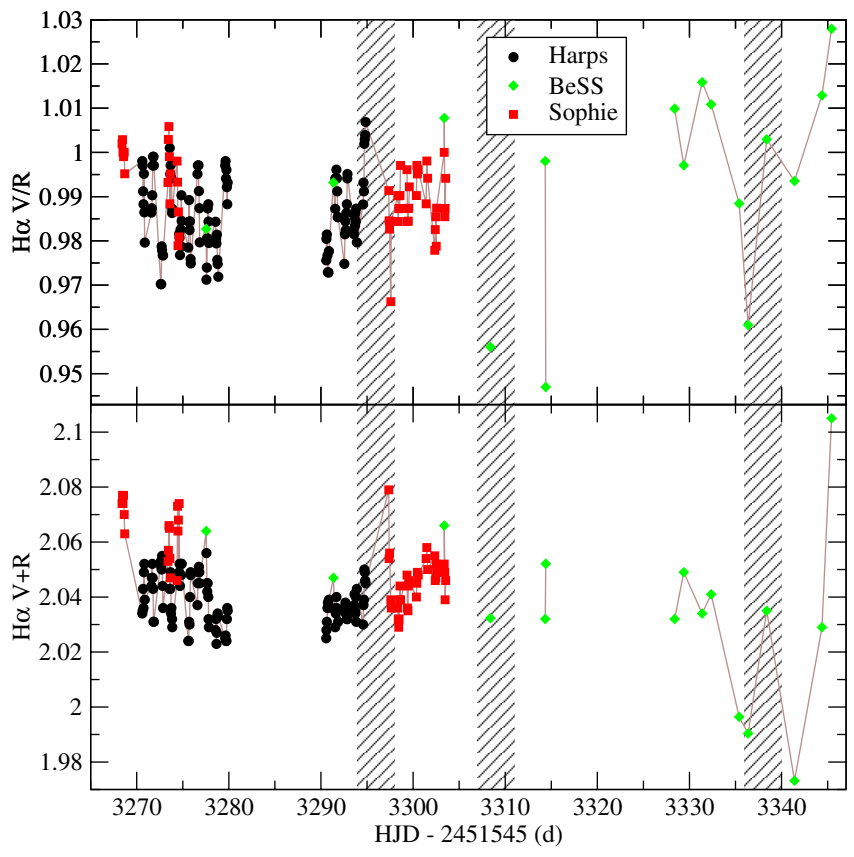

Fig. 3. Violet over red $(V / R)$ emission ratio and sum of the $V$ and $R$ emission measured in the emission peaks of the $\mathrm{H} \alpha$ line observed with HARPS (black circles), SOPHIE (red squares) and from BeSS (green diamonds). The hashed regions indicate the occurrence of the three minor outbursts.

Table 4. The 20 most powerful frequencies (in $\mathrm{c} \mathrm{d}^{-1}$ and $\mu \mathrm{Hz}$ ) detected in the full CoRoT light curve of HD 51452 with the CLEAN-NG method.

\begin{tabular}{ccrcc}
\hline \hline & $\begin{array}{c}\text { Frequency } \\
\mathrm{c} \mathrm{d}^{-1}\end{array}$ & $\mu \mathrm{Hz}$ & $\begin{array}{c}\text { Amplitude } \\
\mu \mathrm{mag}\end{array}$ & $\begin{array}{c}\text { Phase } \\
{[0: 1]}\end{array}$ \\
\hline$f_{1}$ & 0.6293 & 7.284 & $1653.1 \pm 84.9$ & $0.51 \pm 0.01$ \\
$f_{2}$ & 0.0131 & 0.152 & $1292.6 \pm 4.05$ & $0.86 \pm 0.00$ \\
$f_{3}$ & 0.0524 & 0.607 & $1123.0 \pm 4.38$ & $0.13 \pm 0.00$ \\
$f_{4}$ & 0.6315 & 7.309 & $1043.7 \pm 71.5$ & $0.01 \pm 0.01$ \\
$f_{5}$ & 0.0240 & 0.278 & $1042.3 \pm 4.1$ & $0.95 \pm 0.00$ \\
$f_{6}$ & 1.2302 & 14.239 & $945.9 \pm 12.6$ & $0.85 \pm 0.00$ \\
$f_{7}$ & 0.1464 & 1.695 & $778.2 \pm 9.5$ & $0.11 \pm 0.00$ \\
$f_{8}$ & 0.6140 & 7.107 & $758.6 \pm 25.8$ & $0.50 \pm 0.01$ \\
$f_{9}$ & 0.1595 & 1.846 & $742.0 \pm 4.1$ & $0.28 \pm 0.00$ \\
$f_{10}$ & 1.0008 & 11.583 & $704.5 \pm 3.9$ & $0.07 \pm 0.00$ \\
$f_{11}$ & 1.0620 & 12.292 & $682.3 \pm 4.0$ & $0.14 \pm 0.00$ \\
$f_{12}$ & 0.6468 & 7.486 & $674.1 \pm 8.4$ & $0.90 \pm 0.00$ \\
$f_{13}$ & 0.6643 & 7.689 & $652.7 \pm 4.4$ & $0.04 \pm 0.00$ \\
$f_{14}$ & 0.4654 & 5.387 & $648.9 \pm 5.3$ & $0.58 \pm 0.00$ \\
$f_{15}$ & 0.6774 & 7.840 & $643.1 \pm 4.1$ & $0.81 \pm 0.00$ \\
$f_{16}$ & 1.8290 & 21.169 & $640.3 \pm 4.4$ & $0.49 \pm 0.00$ \\
$f_{17}$ & 1.2193 & 14.113 & $629.2 \pm 4.6$ & $0.69 \pm 0.00$ \\
$f_{18}$ & 0.6403 & 7.410 & $622.6 \pm 14.5$ & $0.95 \pm 0.00$ \\
$f_{19}$ & 0.7036 & 8.144 & $621.4 \pm 4.0$ & $0.78 \pm 0.00$ \\
$f_{20}$ & 0.0896 & 1.037 & $616.7 \pm 5.4$ & $0.43 \pm 0.00$ \\
\hline
\end{tabular}

Notes. The amplitudes and phases calculated with AMPHI are reported in Cols. 5 and 6 together with their error bars. The full list of 189 frequencies is available in Table 3.

refer to Gutiérrez-Soto et al. (2009). Frequencies found with this method, their amplitude, and phase, as well as the interpretation of each frequency in terms of stellar signal or combinations, but excluding instrumental frequencies such as the orbital frequency of the satellite, are given in the Table 3 . Table 4 shows a shorter list of the 20 frequencies of strongest amplitude only. The amplitudes and phases, and their errors, obtained for each set of 


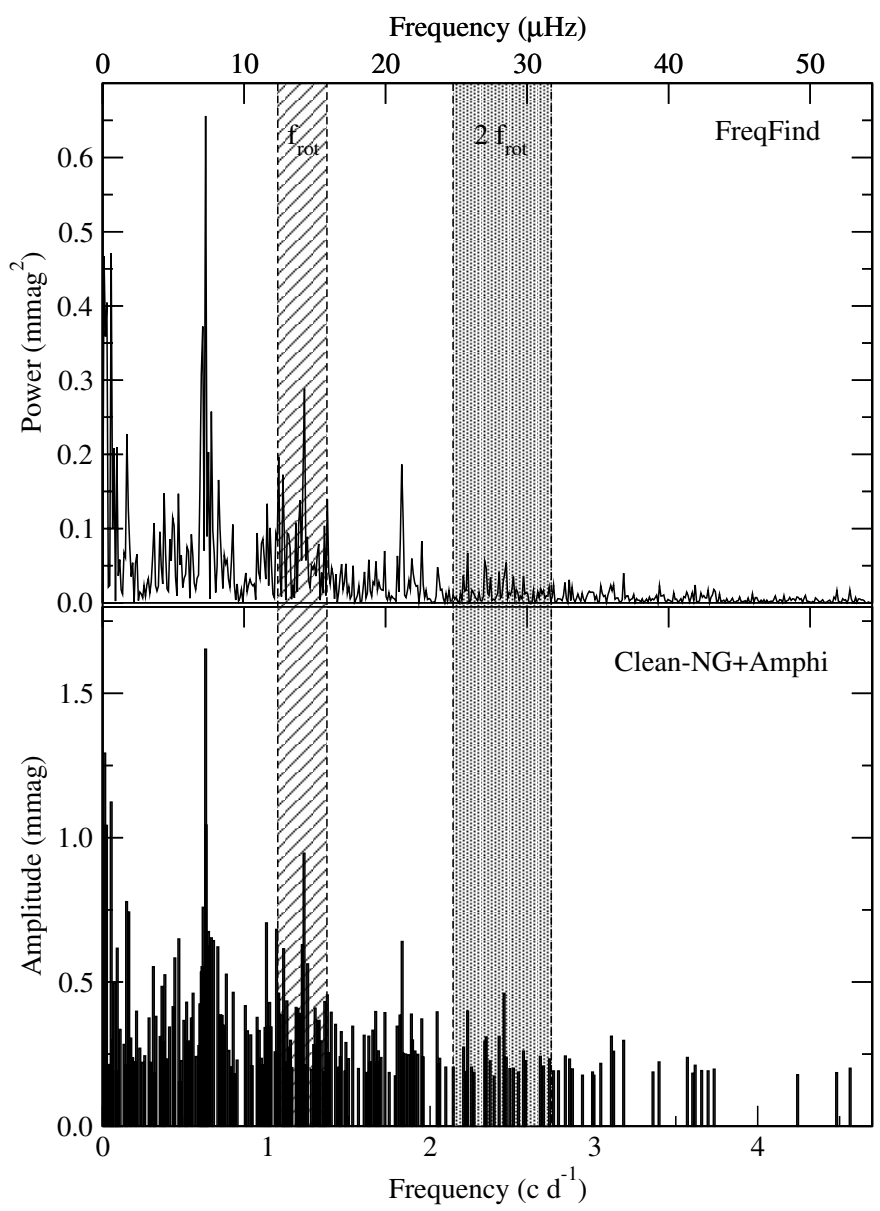

Fig. 4. Power spectrum of the frequencies extracted from the CoRoT data of HD 51452. The hashed and dotted zones represent the error boxes of the rotation frequency and twice this value. Top: Fourier transform from FreQFind. Bottom: Frequencies from Clean-NG with amplitudes recomputed with AMPHI.

frequencies with this method were recalculated with AMPHI to achieve greater accuracy and homogeneity. For a complete set of fixed frequencies, АмРнI calculates the amplitudes and phases to obtain the best fit to the light curve (see Gutiérrez-Soto et al. 2009).

A power spectrum of all significant frequencies obtained with the Clean-NG method is shown in Fig. 4. The Clean-NG method provides 189 frequencies (see Table 3). Some of these frequencies are harmonics or combinations of others, so that the number of independent frequencies is lower than 189. The most outstanding frequencies are the ones forming a regular pattern at $f_{5}=0.0240, f_{4}=0.6315, f_{6}=1.2302, f_{16}=1.8290$, and $f_{33}=$ $2.4517 \mathrm{c} \mathrm{d}^{-1}$. The strong frequency $f_{1}=0.6293$ is equivalent to $f_{4}$ within the error bars on the frequency determination.

We note that $f_{4}, f_{6}$, and $f_{16}$ are perfectly equidistant with $\Delta f=0.5988 \mathrm{c} \mathrm{d}^{-1}$. This frequency $\Delta f$ is not detected directly in the data. The three frequencies probably form a triplet related to a rotational splitting of the pulsation modes. The frequencies $f_{5}$ and $f_{33}$ are not perfectly equidistant to the triplet, since $f_{4}-f_{5}=0.6075 \mathrm{c} \mathrm{d}^{-1}$ and $f_{33}-f_{16}=0.6227 \mathrm{c} \mathrm{d}^{-1}$, which is significantly different from $0.5988 \mathrm{c} \mathrm{d}^{-1}$ with the precision provided by CoRoT. Nevertheless, these two frequency peaks added to the triplet probably form a quasi-equidistant quintuplet, together with $f_{4}, f_{6}$, and $f_{16}$. We recall that the rotation frequency is $f_{\mathrm{rot}} \sim 1.22 \mathrm{c} \mathrm{d}^{-1}$.
Table 5. The 20 most powerful frequencies (in $\mathrm{c} \mathrm{d}^{-1}$ and $\mu \mathrm{Hz}$ ) detected in the full CoRoT light curve of HD 51452 with the FREQFIND method.

\begin{tabular}{lrrlc}
\hline \hline & $\begin{array}{r}\text { Frequency } \\
\mathrm{c} \mathrm{d}^{-1}\end{array}$ & $\mu \mathrm{Hz}$ & \multicolumn{1}{c}{$\begin{array}{c}\text { Amplitude } \\
\mu \mathrm{mag}\end{array}$} & $\begin{array}{c}\text { Phase } \\
{[0: 1]}\end{array}$ \\
\hline$f_{1}^{\prime}=f_{1}$ & 0.6293 & 7.284 & $1170.2 \pm 0.1$ & $0.37 \pm 0.01$ \\
$f_{2}^{\prime}=f_{3}$ & 0.0524 & 0.607 & $973.3 \pm 12.4$ & $0.15 \pm 0.00$ \\
$f_{3}^{\prime}=f_{2}$ & 0.0087 & 0.101 & $983.4 \pm 12.1$ & $0.17 \pm 0.00$ \\
$f_{4}^{\prime}=f_{5}$ & 0.0262 & 0.303 & $898.1 \pm 7.4$ & $0.78 \pm 0.01$ \\
$f_{5}^{\prime}=f_{8}$ & 0.6118 & 7.082 & $861.8 \pm 11.8$ & $0.68 \pm 0.01$ \\
$f_{6}^{\prime}=f_{2}$ & 0.0175 & 0.202 & $833.1 \pm 9.7$ & $0.52 \pm 0.01$ \\
$f_{7}^{\prime}=f_{24}$ & 0.6031 & 6.980 & $782.0 \pm 5.0$ & $0.31 \pm 0.01$ \\
$f_{8}^{\prime}=f_{6}$ & 1.2324 & 14.264 & $774.2 \pm 11.4$ & $0.70 \pm 0.01$ \\
$f_{9}^{\prime}=f_{13}$ & 0.6643 & 7.689 & $734.1 \pm 10.9$ & $0.04 \pm 0.01$ \\
$f_{10}^{\prime}=f_{7}$ & 0.1486 & 1.720 & $688.2 \pm 7.6$ & $0.98 \pm 0.01$ \\
$f_{11}^{\prime}=f_{20}$ & 0.0874 & 1.012 & $648.9 \pm 11.9$ & $0.57 \pm 0.01$ \\
$f_{12}^{\prime}=f_{29}$ & 0.0699 & 0.809 & $637.7 \pm 2.2$ & $0.40 \pm 0.02$ \\
$f_{13}^{\prime}=f_{12}$ & 0.6468 & 7.486 & $632.0 \pm 1.3$ & $0.86 \pm 0.02$ \\
$f_{14}^{\prime}=f_{32}$ & 1.0751 & 12.443 & $631.0 \pm 9.3$ & $0.74 \pm 0.01$ \\
$f_{15}^{\prime}=f_{16}$ & 1.8268 & 21.143 & $610.1 \pm 12.5$ & $0.64 \pm 0.00$ \\
$f_{16}^{\prime}=f_{21}$ & 1.1013 & 12.747 & $594.5 \pm 9.4$ & $0.74 \pm 0.01$ \\
$f_{17}^{\prime}=f_{17}$ & 1.2237 & 14.163 & $577.3 \pm 2.3$ & $0.35 \pm 0.02$ \\
$f_{1,}^{\prime}=f_{19}$ & 0.7080 & 8.194 & $570.6 \pm 11.4$ & $0.56 \pm 0.01$ \\
$f_{19}^{\prime}=f_{14}$ & 0.4633 & 5.362 & $560.8 \pm 10.9$ & $0.71 \pm 0.01$ \\
$f_{20}^{\prime}=f_{35}$ & 1.3723 & 15.883 & $548.4 \pm 7.3$ & $0.47 \pm 0.02$ \\
\hline
\end{tabular}

Notes. The corresponding ClEan-NG frequency number (see Tables 4 and 3) is also indicated in Col. 1.

FREQFIND uses a fast Fourier transform and iterates to find the frequency peaks one by one and remove them from the signal in the time domain. It is described and tested in detail in Leroy et al. (in prep.). It uses a stopping criterion based on the ratio between the frequency peak and the surrounding frequency domain in the residual spectrum. We used a ratio of 3.8 to obtain the 20 strongest frequencies listed in Table 5 and check the results obtained with CLEAN-NG.

FREQFIND provides similar results to CLEAN-NG in terms of frequencies, within the $0.0022 \mathrm{c} \mathrm{d}^{-1}$ uncertainty of each determination. Table 5 shows the correspondance between the main frequencies obtained with FREQFIND and those from CLEAN-NG. The amplitude determined by FREQFIND, however, are significantly different from those calculated with AMPHI on CleanNG frequencies. This discrepancy is certainly related to the fact that we determined only the 20 most powerful frequencies with FREQFIND while we considered the full set of 189 frequencies for Clean-NG.

\subsubsection{Fourier analysis of the light curve pieces between outbursts}

The CoRoT light curve was separated into 4 pieces, cut at the time of minor outbursts. This represents pieces of the light curve of $139482,31361,70950$ and 36265 data points respectively.

The same analysis method as for the full light curve was then applied to the 4 separate pieces of the light curve. Figure 5 shows the 4 resulting power spectra. We found 125, 61, 88 and 69 frequencies respectively with the CLEAN-NG method. The main frequencies are present almost all the time but with very different amplitudes. For example $f_{1}$ dominates during the second and third parts of the light curve, $f_{4}$ during the first part, and $f_{6}$ and $f_{16}$ are stronger in the last part.

In addition, some other frequencies with strong amplitudes appear clearly in some parts, e.g. around $1.1 \mathrm{c} \mathrm{d}^{-1}$ in parts 2 and 4 of the light curve, or close to 0 during the third part. 


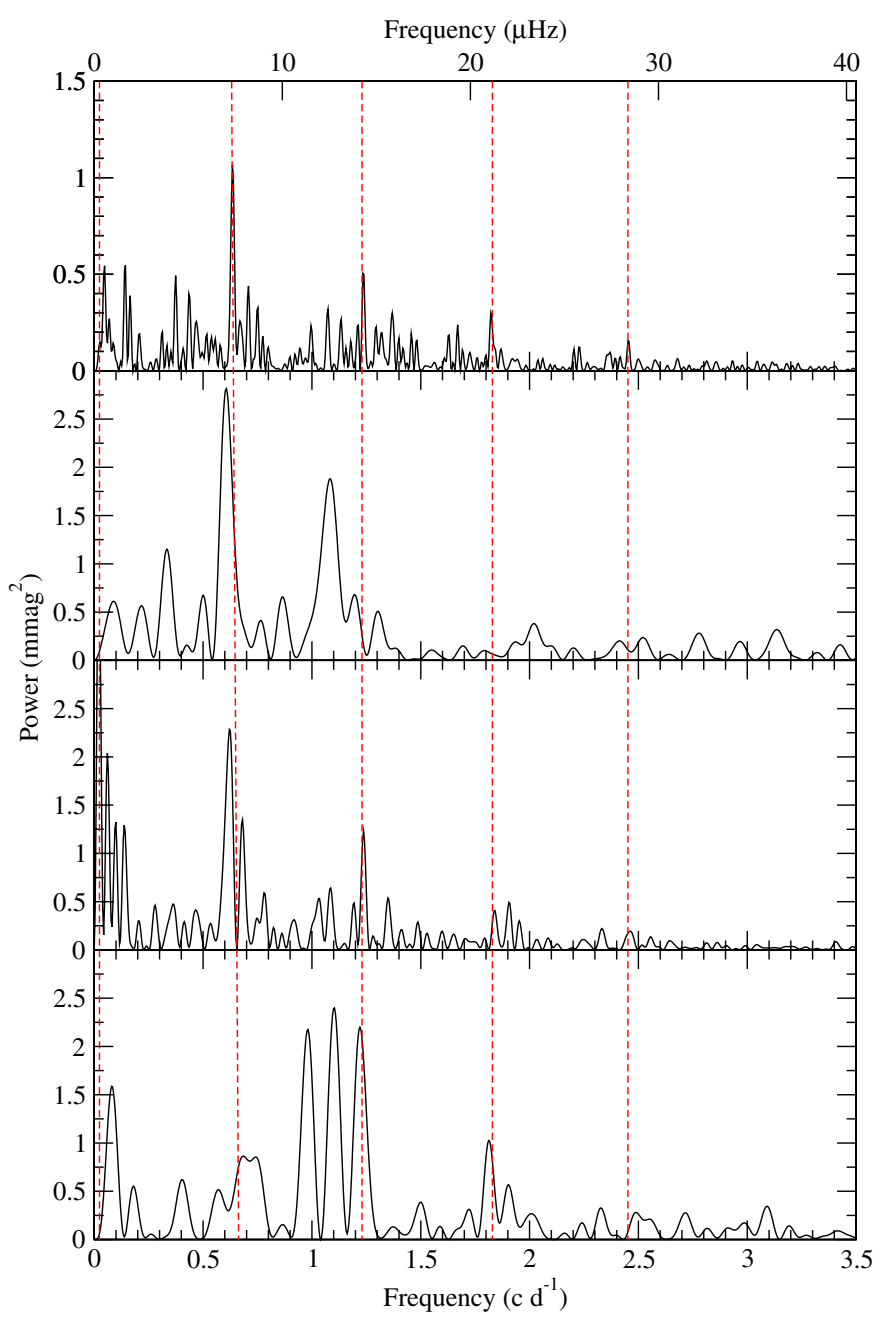

Fig. 5. Fourier transform of the frequencies calculated with FREQFIND extracted from 4 pieces of the CoRoT data: until HJD $=3297$ (top), between HJD $=3297$ and 3310 (second panel), between HJD $=3310$ and 3339 (third panel), and after HJD = 3339 (bottom). We adjusted the oversampling value used in FREQFIND to compensate for the different length of the 4 pieces. The top panel is shown with a y-range divided by 2 compared to the other 3 panels. The red dashed lines indicate the quintuplet centered on $f_{6}$ (see text).

\subsubsection{Time-frequency analysis}

Considering the changing amplitudes observed in Sect. 4.1.2, we performed a time-frequency analysis of the CoRoT data using a sliding window of 30 days, shifted two days by two days, and an oversampling value of 16 . This method is described in detail in Huat et al. (2009). We searched for frequencies with Clean-NG in each subsample. We then recalculated the amplitudes and phases with AmPHI.

A 3D plot of all frequencies between 0 and $2 \mathrm{c} \mathrm{d}^{-1}$ detected with the time-frequency method is shown in the top panel of Fig. 7. When viewed from the top, this plot corresponds to a periodogram. The bottom panel of Fig. 7 shows such a periodogram for the strongest frequencies. We see that the amplitude of the main frequencies varied with time during the CoRoT run. In particular frequency $f_{4}$ had a larger amplitude before the small outburst at HJD $-2452545=3297$, while frequencies $f_{1}$ and $f_{6}$ were stronger after that date.

Amplitude variations have been found in other Be stars observed with CoRoT, e.g. in HD 181231 (Neiner et al. 2009) and HD 49330 (Huat et al. 2009).

\subsection{Ground-based data}

\subsubsection{Line-profile variations}

The inspection of spectral lines in the HARPS and SOPHIE data indicated that variation occurred with time, especially line splitting. A time series analysis was performed on the ground-based HARPS and SOPHIE data using Fourier transform and least squares fitting methods, as in Floquet et al. (2002) and Neiner et al. (2005). We first calculated residual spectra by removing the mean profile from each spectrum. The time series analysis was then applied to each wavelength bin of the time series of the residual spectra to study the line profile variations (lpv) of the $\mathrm{He}_{\mathrm{I}} 4471,4922,5015,6678, \mathrm{Mg}$ II 4481, and $\mathrm{H} \beta$ lines. The minimum detectable frequency was $1 / T=0.03 \mathrm{c} \mathrm{d}^{-1}$, i.e., $0.35 \mu \mathrm{Hz}$, where $T$ is the length of the HARPS + SOPHIE run. The error bar of the frequencies is $1 /(4 T)=0.008 \mathrm{c} \mathrm{d}^{-1}$, i.e., $0.09 \mu \mathrm{Hz}$.

For all studied lines, we observed variations in the data but significant frequencies were hard to extract. Although we did obtain six or seven spectra per night with HARPS and SOPHIE, this could only be done over $\sim 10$ consecutive days and with relatively poor signal-to-noise, which was not enough to detect frequencies with low amplitudes. We thus imposed the frequencies detected in the CoRoT data to the residual spectra and checked if coherent variations could be found.

For all studied lines, we found that the main frequency $f_{4}$ produced a relatively coherent variation showing bumps travelling from blue to red and from red to blue. An example of a dynamical spectrum folded with this frequency is given in Fig. 8 for the $\mathrm{H} \beta$ line. This plot shows crossing patterns. This indicates that the inclination angle $i$ allowed us to see on both sides of the rotation axis, which is compatible with the derived value of $i=[39-59]^{\circ}$ (see Sect. 3.1).

Following Telting \& Schrijvers (1997), it is possible to determine the $l$ degree of a pulsation mode from its power and phase diagrams: the slope of the phase diagram in the region where the amplitude is significant is directly related to $l$. The distribution in power and phase of $f_{4}$ (see Fig. 9) allowed us to estimate a value of the $l$ degree of about 10 . Since HD 51452 rotates very fast, this corresponds to the dominant $l$ degree at the considered frequency.

Frequencies $f_{6}$ and $f_{16}$ were also found to have a coherent variation in the line profiles. No clear determination of the $l$ degree could be obtained from the distribution in power and phase for $f_{6}$. For $f_{16}$, the dominant $l$ degree is lower than for $f_{4}$ and equals about 4 (see Fig. 10).

\subsubsection{Variations in spectral quantities}

The time series analysis was also performed on spectral quantities such as the $V / R$ emission ratio of the $\mathrm{H} \alpha$ line. We obtained frequencies at $F_{1}=0.666, F_{2}=1.61, F_{3}=0.269, F_{4}=1.052$ and $F_{5}=1.069 \mathrm{c} \mathrm{d}^{-1}$. Taking precision and 1-day aliases into account, these frequencies all correspond to frequencies found in the CoRoT data: $F_{1} \sim f_{4}, F_{2} \sim f_{1}+1, F_{3} \sim f_{6}-1, F_{4}$ and $F_{5} \sim f_{3}+1$.

\section{Discussion}

\subsection{Frequencies and amplitudes of the pulsation modes}

We showed that the HD 51452 displays many frequencies of variations associated with pulsations. The amplitude of the main frequencies of pulsations varies with time and notably as minor outbursts occur. Since $f_{\text {rot }}=1.22 \pm 0.15 \mathrm{c} \mathrm{d}^{-1}$, the detected 


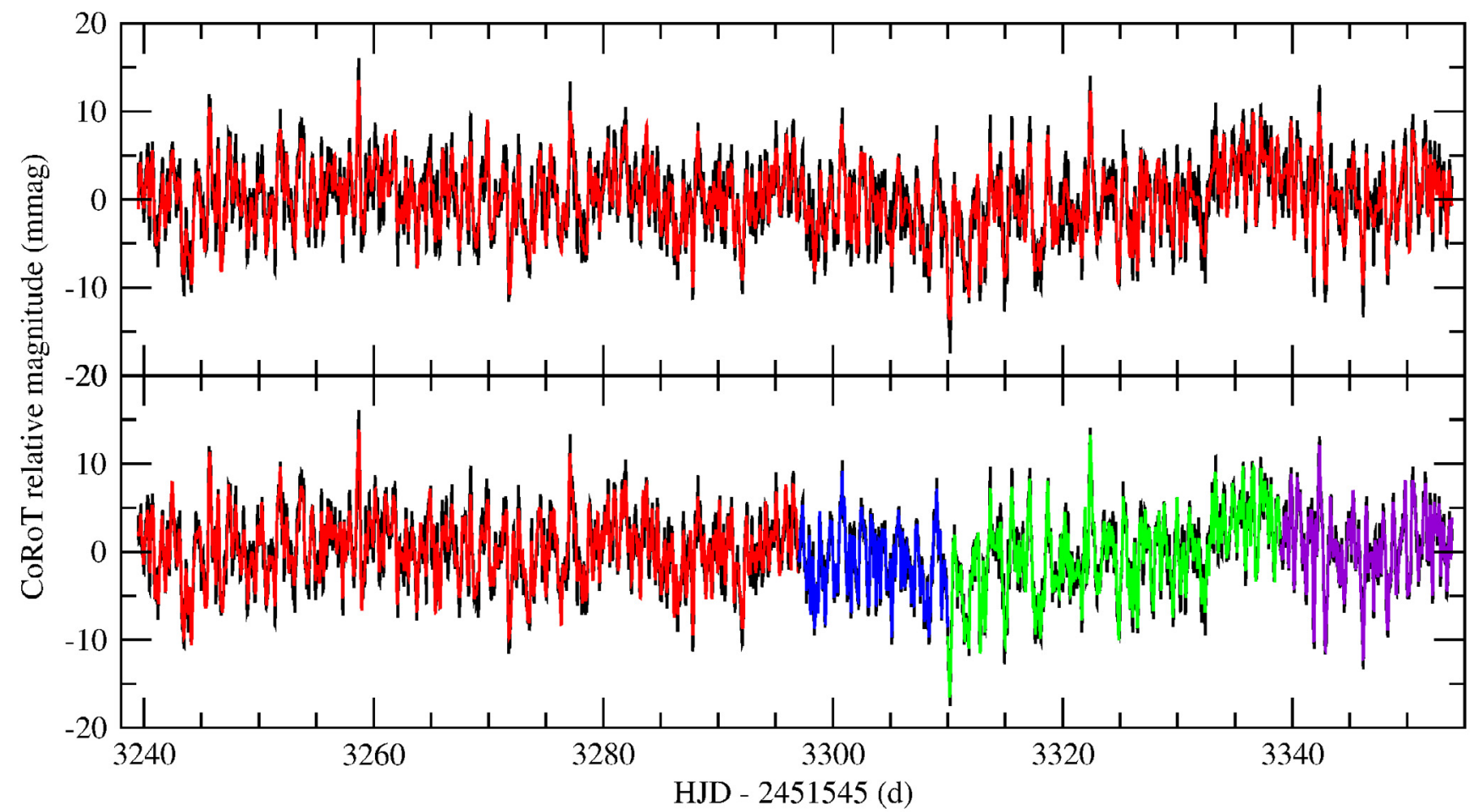

Fig. 6. Model of the CoRoT light curve (in colors) computed with the frequencies, amplitudes, and phases determined with CLEAN-NG and AMPHI, superimposed on the observed CoRoT light curve (in black). Top: the full light curve fitted with 189 frequencies, bottom: the light curve in 4 pieces fitted with 125, 61, 88, and 69 frequencies.
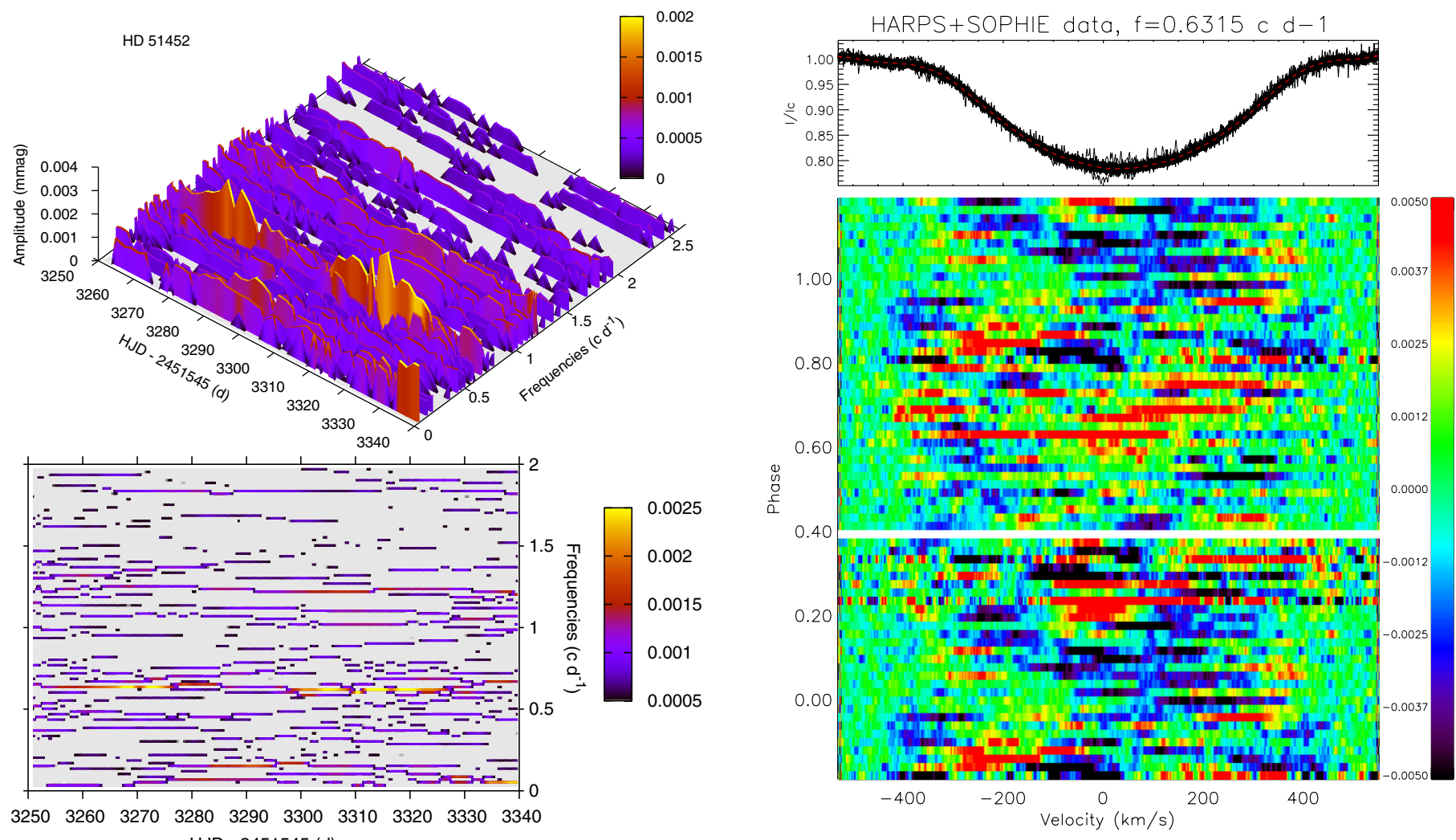

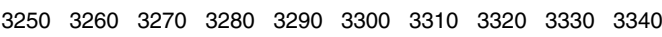
HJD - 2451545 (d)

Fig. 7. 3D plot (top panel) and periodogram (bottom panel) of the results of the time-frequency analysis of the CoRoT light curve of HD 51452. In the bottom panel, the frequencies with the lowest amplitudes were truncated to make the plot clearer.

Fig. 8. Top panel: HARPS and SOPHIE observations of the $\mathrm{H} \beta$ line. The dashed red line is the mean profile. Bottom panel: dynamical spectrum of the residuals of the $\mathrm{H} \beta$ line from the mean profile, folded in phase with $f_{4}=0.6315 \mathrm{c} \mathrm{d}^{-1}$. 

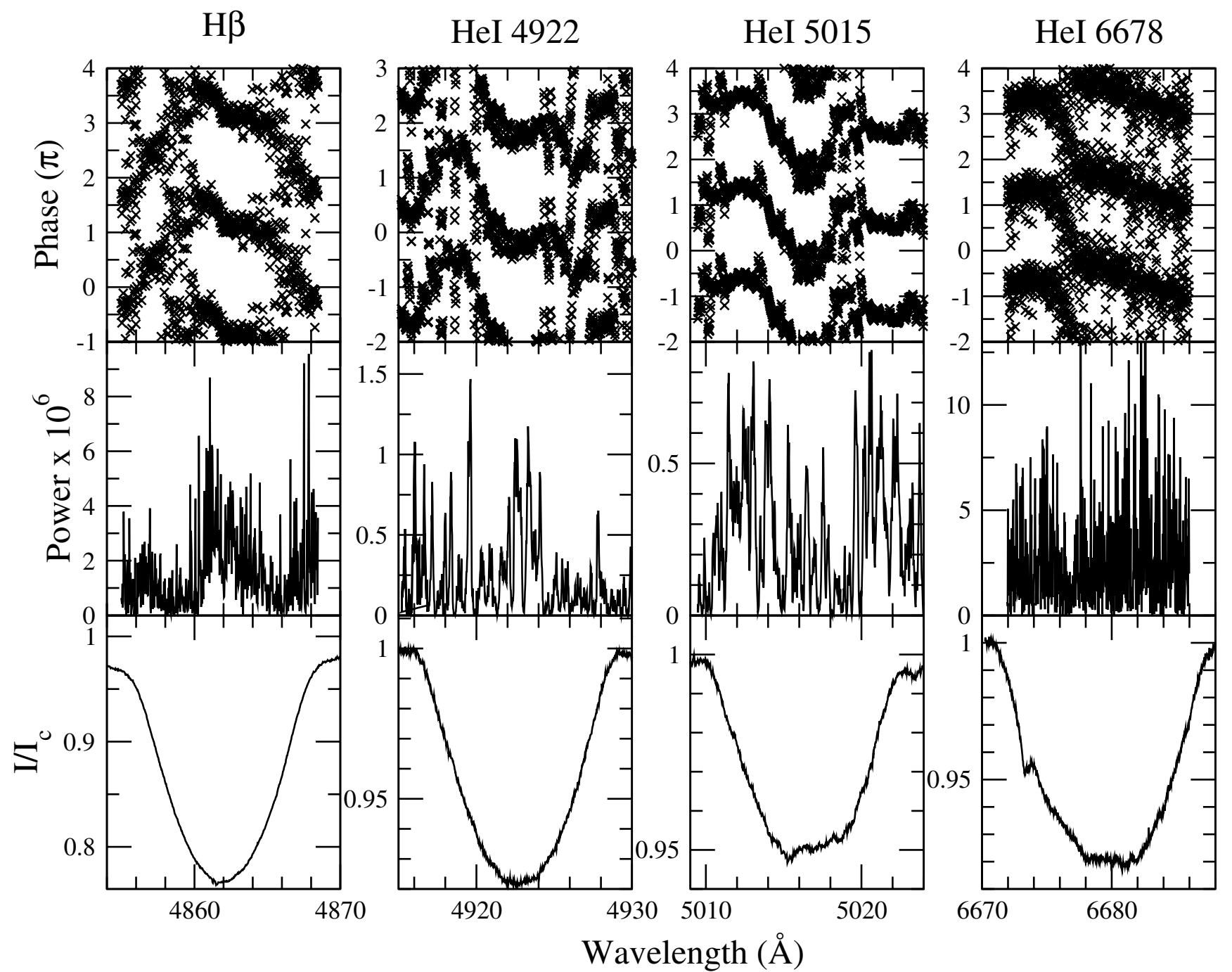

Fig. 9. Phase (top panel) and power (middle panel) of the frequency $f_{4}=0.6315 \mathrm{c} \mathrm{d}^{-1}$ along the mean SOPHIE+HARPS line profile (bottom panel) for the $\mathrm{H} \beta, \mathrm{He}_{\mathrm{I}} 4922,5015$, and 6678 lines.

frequencies are all below $2 \times f_{\text {rot }}$; i.e., they are gravito-inertial (gi) modes, in the subinertial frequency range where the Coriolis acceleration has a strong effect. Gravito-inertial modes are simultaneously driven by the buoyancy force and the Coriolis acceleration.

Frequencies $f_{5}, f_{4}, f_{6}, f_{16}$, and $f_{33}$ may form a quintuplet. Pulsation r-modes, which constitutes a subclass of gi modes mainly driven by the Coriolis acceleration, with the same $l$ and different $m$ are indeed expected to form pattern with equidistant frequency peaks, to the first order of rotational effects. That $f_{5}$ and $f_{33}$ are not perfectly equidistant to the other three peaks might be a higher effect of fast rotation (Provost et al. 1981). In addition, differential rotation, especially radially, could modify the frequencies of the modes.

One of the main frequency, $f_{6}=1.2302 \mathrm{c} \mathrm{d}^{-1}$, falls in the range of possible rotation frequency. However, this is one of the peaks of the multiplet mentioned above. It is not the strongest peak and the rest of the multiplet does not correspond to harmonics of this peak. Therefore this multiplet cannot be attributed to rotational modulation. However, any other lower peak detected in the frequency domain $1.22 \pm 0.15 \mathrm{c} \mathrm{d}^{-1}$ could be the rotation frequency.

\subsection{Origin of the observed frequencies}

HD 51452 is a hot Be star as shown by Frémat et al. (2006) and in Sect. 3.1. For such a hot star, the $\kappa$-mechanism can create p-modes of pulsations or possibly low-order g-modes with frequencies above $\sim 1.5 \mathrm{c} \mathrm{d}^{-1}$. Nevertheless, g-modes are detected in HD 51452 with frequencies below $\sim 1.5 \mathrm{c} \mathrm{d}^{-1}$. We thus investigate below how this is possible.

\subsection{1. $\kappa$-driven modes thanks to stellar flattening}

Since HD 51452 rotates close to its critical velocity, at which the centrifugal force balances gravitation at the equator, the star is very flattened, and its temperature is very different at the poles and at the equator. One possible explanation could therefore be that the temperature in the equatorial region is low enough to allow the excitation of $g$-modes by the $\kappa$-mechanism. This is particularly appealing since g-modes are known to be trapped in the equatorial region in rapidly rotating stars.

The inclination angle at which we observe the star is around $\sim 45$ degrees, and the observer thus does clearly see both one pole and the equator of the star. Accordingly, the spectra 

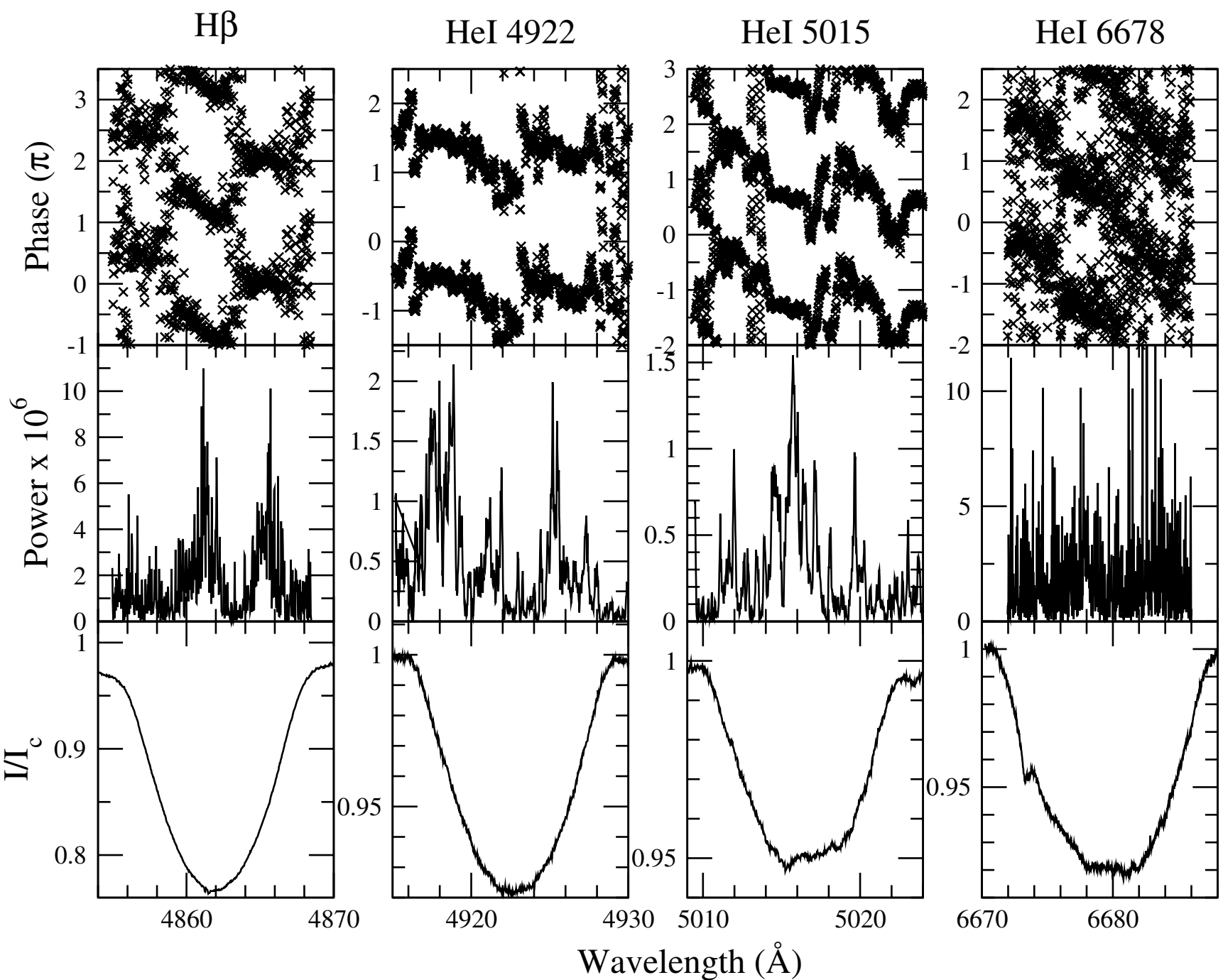

Fig. 10. Same as Fig. 9 but for frequency $f_{16}=1.8290 \mathrm{c} \mathrm{d}^{-1}$.

reflect the average temperature at the stellar surface. A difference of at least $11000 \mathrm{~K}$ or more would be required between this averaged temperature presented in Sect. $3.1(31000 \mathrm{~K})$ and the temperature at the equator, for the latter to be cool enough (20000 K, Pamyatnykh 1999) to host g-modes driven by the $\kappa$-mechanism.

We calculated the gradient of effective temperature at the surface of a rotating star by following the results of Espinosa Lara \& Rieutord (2011). We found that, with $\Omega / \Omega_{\mathrm{c}}=0.9$, the difference in temperature between the poles and the equator is only $5000 \mathrm{~K}$. Even pushing to $\Omega / \Omega_{\mathrm{c}}=0.99$ and using the averaged temperature determined from the spectra as the polar temperature rather than the averaged temperature, we find a temperature difference of only $9000 \mathrm{~K}$ with an equatorial temperature of $22000 \mathrm{~K}$, i.e. too high to allow the excitation of g-modes by the $\kappa$-mechanism. See Fig. 11.

Therefore stellar flattening does not allow a strong enough temperature gradient to explain the presence of g-modes in HD 51452 with the $\kappa$-mechanism.

\subsection{2. $\kappa$-driven modes thanks to enhanced metallicity}

Another possibility that one can consider to excite g-modes in a star as hot as HD 51452 with the $\kappa$-mechanism is the

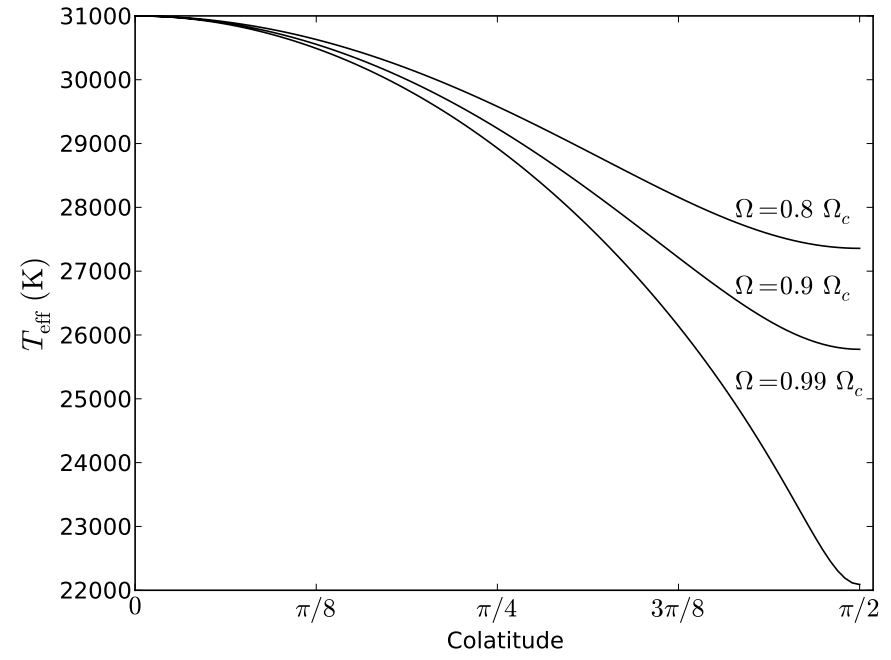

Fig. 11. Gradient of effective temperature at the surface of a rotating star, calculated following Espinosa Lara \& Rieutord (2011).

change of metallicity compared to solar metallicity. Indeed the $\kappa$-mechanism is connected with the opacity in the Fe or Ni ionization zones (Salmon et al. 2012). A higher and larger opacity 


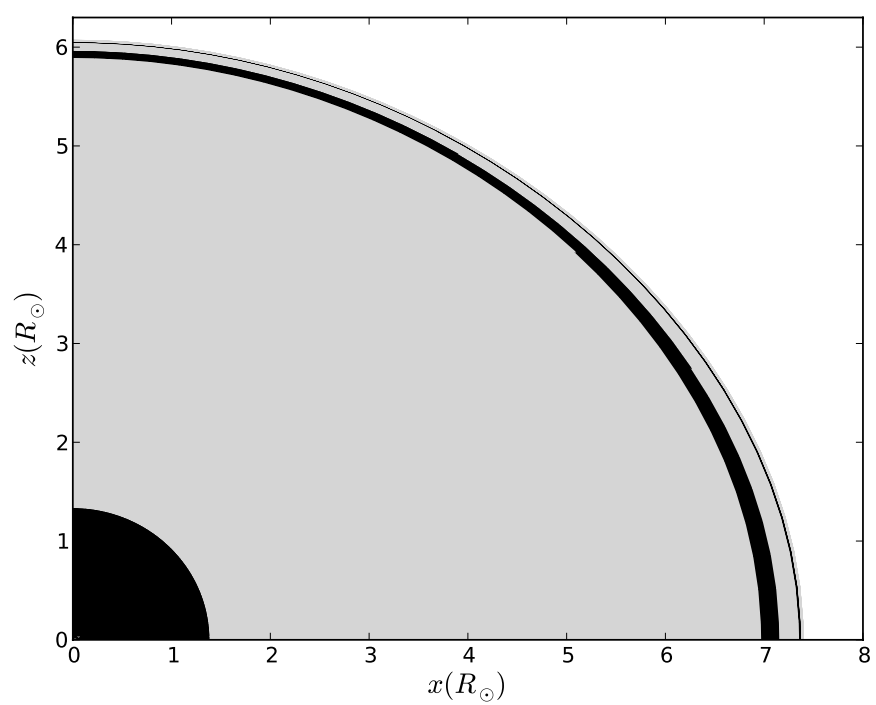

Fig. 12. A quarter of the star HD 51452 modeled with the ESTER code. Convective zones are indicated in black.

peak implies a larger instability strip and a larger frequency domain of excited modes (Montalbán et al. 2009).

However, in Sect. 3.1 we showed that the spectra of HD 51452 are better fit with a solar metallicity than when doubling the abundances of the main chemical elements including Fe. Thus it seems that the metallicity of HD 51452 is close to solar. Actually, Morel (2009) showed that the element abundances in nearby OB stars are usually lower than the solar abundances.

As done for some hot $\beta$ Cep stars (e.g. $v$ Eri, Pamyatnykh et al. 2004), one could alternatively invoke an ad hoc local enhancement of iron group elements in the driving zone, not visible at the surface. However, no evidence is available to justify this speculation.

\subsubsection{Stochastically excited gravito-inertial modes}

The frequencies below $\sim 1.5 \mathrm{c} \mathrm{d}^{-1}$ detected in the CoRoT data of HD 51452 should thus be attributed to another excitation mechanism than the $\kappa$-mechanism. Stochastic low $n$-order gi-modes can be excited in the convective core of Be stars while asymptotic gi-modes can be excited by the iron ionization opacity peak in the subsurface convection zones (Belkacem et al. 2010; Samadi et al. 2010, see also Cantiello et al. 2011).

We computed a model with the ESTER code (e.g. Espinosa Lara \& Rieutord 2007; Rieutord \& Espinosa Lara 2009; Espinosa Lara 2010). ESTER calculates the 2D structure of a rotating star without stellar evolution. We used the stellar parameters published in Frémat et al. (2006) for $\Omega / \Omega_{\mathrm{c}}=0.9$. The stellar mass was fixed to $15.8 M_{\odot}$ and the inclination angle to $46^{\circ}$. We then adjusted the abundance of hydrogen in the stellar core to obtain the values of $T_{\text {eff }}, \log g$ and $v \sin i$ from Frémat et al. (2006). The chemical abundance in the star is homogeneous except for hydrogen, which in the core is $70 \%$ of the abundance in the envelope. We introduced no overshoot.

Figure 12 shows a quarter of the star with convective zones shown in black. We see that, in addition to the convective core, a convective layer develops just below the surface. The location of this convective zone in the envelope is related to the opacity distribution in the star; i.e., its location is where convection is needed to transport energy outwards. This convective layer is slightly thicker at the equator than at the pole, owing to the flattened shape of the star. Since the amplitude of stochastically excited modes is related to the volume of the convective zone, the thickness of the convective zone influences the amplitudes of the modes. Theoretical calculations of stochastic excitation can be found in Belkacem et al. (2009) and Samadi et al. (2010), and more precise calculations applied to HD 51452 will be the subject of a forthcoming paper (see also Sect. 5.3).

Stochastic gi-modes thus appear as a realistic explanation for the presence of low frequencies in HD 51452, in particular subinertial gi-modes would be below $\sim 2.44 \mathrm{c} \mathrm{d}^{-1}$. There are two types of gi-modes: (1) those usually called g-modes, which are gravity modes modified by the Coriolis acceleration and which show a regular pattern in period when they are asymptotic (Lee \& Saio 1997; Ballot et al. 2010), and (2) r-modes, which are mainly driven by the Coriolis acceleration, are subinertial and show regular patterns in frequency (Provost et al. 1981; Saio 1982; Lee 2006).

Since we observed at least one triplet/quintuplet in frequency in the subinertial domain in the power spectrum of the CoRoT data of HD 51452, those peaks can be interpreted as r-modes. Since we observe no specific frequency or period spacing for the other frequency peaks, they could be any type of gi-modes.

Stochastic waves are also put forward as an explanation (Neiner et al., in prep.) for the transport of angular momentum in the outbursting Be star HD 49330 observed with CoRoT (Huat et al. 2009; Floquet et al. 2009).

\subsection{Excitation of gravito-inertial modes}

Samadi et al. (2010) examined the stochastic excitation of gravity modes by turbulent convection in massive stars. They computed the power supplied by turbulent eddies to the modes in the stellar core as well as in the outer convective zones. They found that the excitation of low $n$-order g-modes occurs in the core while the asymptotic g-modes are mostly excited in the outer convective zone. Such excitation in the core has also been identified in numerical simulations (Browning et al. 2004). The mode amplitudes that Samadi et al. (2010) deduced, however, are well below the detection threshold of the CoRoT satellite for a massive star.

In the work by Samadi et al. (2010), no rotation is considered. In Belkacem et al. (2009), the influence of the Coriolis acceleration on the stochastic excitation of modes by convective zones has been examined. They found that the influence of rotation as a direct contribution to mode driving is negligible, but that excitation-rate asymmetries between prograde and retrograde modes appear. In their paper, however, they only applied their formalism to slowly uniformly rotating solar-type stars.

HD 51452 is a Be star, rotating close to its breakup velocity; therefore, the calculation of the excitation of gi-modes (including r-modes) in this star would require the study of the influence of very fast rotation in the convective zones of a massive star. This application can be derived from the work by Belkacem et al. (2009) and will be the study of a forthcoming paper. The detection of gi-modes in HD 51452 presented here, however, suggests that fast rotation enhances the amplitude of gi-modes, hence r-modes.

\section{Conclusions}

Using time series of almost four months of high-precision high-cadence photometric CoRoT observations, we detected many frequencies of variations in HD 51452. Thanks to groundbased spectroscopic data obtained during the same period, we found that the $l$ order of the main modes are rather large 
(4 to 10 depending on the frequency). Considering the temperature of the star and the observed frequencies, the detected modes cannot be attributed to the $\kappa$-mechanism.

For the first time we have shown that a hot Be star, HD 51452, hosts gravito-inertial modes of pulsations, including r-modes, that are probably stochastically excited in the convective core or possibly in the outer convective zone of the star. Theory has not predicted these modes to be detectable with CoRoT. The reason their amplitude is above the detection threshold could be related to the very rapid rotation of the star.

That HD 51452 is a very hot Be star excludes the possibility that the observed gi modes are excited by the $\kappa$-mechanism. For other cooler B or Be stars, the $\kappa$-mechanism seemed like an obvious excitation mechanism. In view of the results presented here, however, it might be necessary to reconsider our interpretation of several other rapidly rotating B or Be stars, for which stochastic excitation might also be at work; for example, the recent observation of gi modes in HD 43317 by Pápics et al. (2012) with CoRoT could possibly also be due to stochastic excitation.

In the same way, the puzzling detection of high-order gmodes in a few hot $\beta$ Cep stars, such as $v$ Eri (Handler et al. 2004) or 12 Lac (Handler et al. 2006), might be reinvestigated in the frame of stochastic excitation. For these stars a very high and unrealistic increase of iron abundance in the driving zone is indeed necessary to explain the observed pulsation frequencies with the $\kappa$-mechanism. Stochastic excitation might solve this problem; however, these $\beta$ Cep stars rotate much more slowly than Be stars.

Finally, the amplitude of the observed r-modes is linked to the occurrence of minor outbursts. This is additional observational evidence of the relation between pulsations and the $\mathrm{Be}$ phenomenon already put forward by Huat et al. (2009). See also the theoretical work of Lee (2006) on the transport of angular momentum by r-modes.

Acknowledgements. We wish to thank the CoRoT team for the acquisition and reduction of the CoRoT data. C.N. thanks Rafael Garcia for useful discussions. C.N. thanks CNES and ANR (SIROCO) for their financial support. E.P. and M.R. acknowledge financial support from the Italian PRIN-INAF 2010 Asterosesismology: looking inside the stars with space- and ground-based observations. The work of P.D. and J.G.S. is supported by the Spanish Programa Nacional de Astronomía y Astrofísica under contracts AYA2010-18352 and AYA2010-20982-C02-01, respectively. M.B. is an F.R.S.-FNRS Postdoctoral Researcher, Belgium. This work has made use of the BeSS database, operated at LESIA, Observatoire de Meudon, France: http://basebe.obspm.fr.

\section{References}

Auvergne, M., Bodin, P., Boisnard, L., et al. 2009, A\&A, 506, 411

Ballot, J., Lignières, F., Reese, D. R., \& Rieutord, M. 2010, A\&A, 518, A30

Belkacem, K., Mathis, S., Goupil, M. J., \& Samadi, R. 2009, A\&A, 508, 345

Belkacem, K., Dupret, M. A., \& Noels, A. 2010, A\&A, 510, A6

Browning, M. K., Brun, A. S., \& Toomre, J. 2004, ApJ, 601, 512

Cantiello, M., Braithwaite, J., Brandenburg, A., et al. 2011, in IAU Symp., 272, eds. C. Neiner, G. Wade, G. Meynet, \& G. Peters, 32

Dziembowski, W. A., \& Pamyatnykh, A. A. 1993, MNRAS, 262, 204

Dziembowski, W. A., Moskalik, P., \& Pamyatnykh, A. A. 1993, MNRAS, 265, 588

Espinosa Lara, F. 2010, Ap\&SS, 328, 291

Espinosa Lara, F., \& Rieutord, M. 2007, A\&A, 470, 1013

Espinosa Lara, F., \& Rieutord, M. 2011, A\&A, 533, A43

Floquet, M., Neiner, C., Janot-Pacheco, E., et al. 2002, A\&A, 394, 137

Floquet, M., Hubert, A.-M., Huat, A.-L., et al. 2009, A\&A, 506, 103

Frémat, Y., Neiner, C., Hubert, A.-M., et al. 2006, A\&A, 451, 1053

Gutiérrez-Soto, J., Floquet, M., Samadi, R., et al. 2009, A\&A, 506, 133

Handler, G., Aerts, C., \& An International Team Of 50 Astronomers 2004, in Variable Stars in the Local Group, eds. D. W. Kurtz, \& K. R. Pollard, IAU Colloq. 193, ASP Conf. Ser., 310, 221

Handler, G., Jerzykiewicz, M., Rodríguez, E., et al. 2006, MNRAS, 365, 327

Huat, A.-L., Hubert, A.-M., Baudin, F., et al. 2009, A\&A, 506, 95

Kallinger, T., Reegen, P., \& Weiss, W. W. 2008, A\&A, 481, 571

Lee, U. 2006, MNRAS, 365, 677

Lee, U., \& Saio, H. 1997, ApJ, 491, 839

Martins, F. 2011, Bull. Soc. Roy. Sci. Liège, 80, 29

Montalbán, J., Miglio, A., \& Morel, T. 2009, Commun. Asteros., 158, 288

Morel, T. 2009, Commun. Asteros., 158, 122

Neiner, C., Floquet, M., Hubert, A. M., et al. 2005, A\&A, 437, 257

Neiner, C., Gutiérrez-Soto, J., Baudin, F., et al. 2009, A\&A, 506, 143

Neiner, C., de Batz, B., Cochard, F., et al. 2011a, AJ, 142, 149

Neiner, C., Wade, G., Meynet, G., \& Peters, G. 2011b, Active OB Stars: Structure, Evolution, Mass Loss, and Critical Limits, IAU Symp., 272

Pamyatnykh, A. A. 1999, Acta Astron., 49, 119

Pamyatnykh, A. A., Handler, G., \& Dziembowski, W. A. 2004, MNRAS, 350 1022

Pápics, P. I., Briquet, M., Baglin, A., et al. 2012, A\&A, 542, A55

Porter, J. M., \& Rivinius, T. 2003, PASP, 115, 1153

Provost, J., Berthomieu, G., \& Rocca, A. 1981, A\&A, 94, 126

Rainer, M. 2003, Master's Thesis, Laurea Thesis, Università degli Studi di Milano

Rieutord, M., \& Espinosa Lara, F. 2009, Commun. Asteros., 158, 99

Saio, H. 1982, ApJ, 256, 717

Salmon, S., Montalbán, J., Morel, T., et al. 2012, MNRAS, 422, 3460

Samadi, R., Fialho, F., Costa, J. E. S., et al. 2007 [arXiv: astro-ph/0703354]

Samadi, R., Belkacem, K., Goupil, M. J., et al. 2010, Ap\&SS, 328, 253

Telting, J. H., \& Schrijvers, C. 1997, A\&A, 317, 723

Wildi, F., Pepe, F., Chazelas, B., Lo Curto, G., \& Lovis, C. 2011, in SPIE Conf. Ser., 8151,47 
Table 3. Frequencies (in $\mathrm{c} \mathrm{d}^{-1}$ and $\mu \mathrm{Hz}$ ) detected in the CoRoT data of HD 51452 with the Clean-NG method.

\begin{tabular}{|c|c|c|c|c|c|}
\hline & $\begin{array}{l}\text { Frequency } \\
\mathrm{c} \mathrm{d}^{-1}\end{array}$ & $\mu \mathrm{Hz}$ & $\begin{array}{c}\text { Amplitude } \\
\mu \mathrm{mag}\end{array}$ & $\begin{array}{c}\text { Phase } \\
{[0: 1]}\end{array}$ & \\
\hline$f_{1}$ & 0.6293 & 7.284 & $1653.1 \pm 84.9$ & $0.51 \pm 0.01$ & $=f_{4},=f_{12}-\delta f(\delta f=0.0175)$ \\
\hline$f_{2}$ & 0.0131 & 0.152 & $1292.6 \pm 4.05$ & $0.86 \pm 0.00$ & \\
\hline$f_{3}$ & 0.0524 & 0.607 & $1123.0 \pm 4.38$ & $0.13 \pm 0.00$ & $=f_{2} * 4$ \\
\hline$f_{4}$ & 0.6315 & 7.309 & $1043.7 \pm 71.5$ & $0.01 \pm 0.01$ & $=f_{1},=f_{6}-\Delta f(\Delta f=0.5988)$ \\
\hline$f_{5}$ & 0.0240 & 0.278 & $1042.3 \pm 4.1$ & $0.95 \pm 0.00$ & $=f_{2} * 2, \sim f_{6}-2 \Delta f$ \\
\hline$f_{6}$ & 1.2302 & 14.239 & $945.9 \pm 12.6$ & $0.85 \pm 0.00$ & $f_{6}$ \\
\hline$f_{7}$ & 0.1464 & 1.695 & $778.2 \pm 9.5$ & $0.11 \pm 0.00$ & \\
\hline$f_{8}$ & 0.6140 & 7.107 & $758.6 \pm 25.8$ & $0.50 \pm 0.01$ & $=f_{6} / 2$ \\
\hline$f_{9}$ & 0.1595 & 1.846 & $742.0 \pm 4.1$ & $0.28 \pm 0.00$ & $=f_{7}+f_{2}$ \\
\hline$f_{10}$ & 1.0008 & 11.583 & $704.5 \pm 3.9$ & $0.07 \pm 0.00$ & \\
\hline$f_{11}$ & 1.0620 & 12.292 & $682.3 \pm 4.0$ & $0.14 \pm 0.00$ & \\
\hline$f_{12}$ & 0.6468 & 7.486 & $674.1 \pm 8.4$ & $0.90 \pm 0.00$ & $=f_{4}+f_{2}$ \\
\hline$f_{13}$ & 0.6643 & 7.689 & $652.7 \pm 4.4$ & $0.04 \pm 0.00$ & $=f_{12}+\delta f,=f_{4}+f_{12}$ \\
\hline$f_{14}$ & 0.4654 & 5.387 & $648.9 \pm 5.3$ & $0.58 \pm 0.00$ & \\
\hline$f_{15}$ & 0.6774 & 7.840 & $643.1 \pm 4.1$ & $0.81 \pm 0.00$ & $=f_{13}+f_{2}$ \\
\hline$f_{16}$ & 1.8290 & 21.169 & $640.3 \pm 4.4$ & $0.49 \pm 0.00$ & $=f_{6}+\Delta f$ \\
\hline$f_{17}$ & 1.2193 & 14.113 & $629.2 \pm 4.6$ & $0.69 \pm 0.00$ & $=f_{9}+f_{11}$ \\
\hline$f_{18}$ & 0.6403 & 7.410 & $622.6 \pm 14.5$ & $0.95 \pm 0.00$ & $=f_{9} * 4$ \\
\hline$f_{19}$ & 0.7036 & 8.144 & $621.4 \pm 4.0$ & $0.78 \pm 0.00$ & $=f_{3}+f_{12},=f_{5}+f_{15}$ \\
\hline$f_{20}$ & 0.0896 & 1.037 & $616.7 \pm 5.4$ & $0.43 \pm 0.00$ & \\
\hline$f_{21}$ & 1.1057 & 12.797 & $614.7 \pm 4.2$ & $0.44 \pm 0.00$ & $=f_{14}+f_{18}$ \\
\hline$f_{22}$ & 0.4414 & 5.109 & $582.9 \pm 4.0$ & $0.67 \pm 0.00$ & $=f_{7} * 3$ \\
\hline$f_{23}$ & 1.2521 & 14.492 & $562.8 \pm 4.1$ & $0.13 \pm 0.00$ & $=f_{1} * 2,=f_{5}+f_{6},=f_{7}+f_{21},=f_{8}+f_{18}$ \\
\hline$f_{24}$ & 0.6075 & 7.031 & $552.8 \pm 33.0$ & $0.99 \pm 0.01$ & $=f_{7}+f_{14}$ \\
\hline$f_{25}$ & 0.3103 & 3.591 & $552.6 \pm 4.3$ & $0.40 \pm 0.00$ & \\
\hline$f_{26}$ & 0.6031 & 6.980 & $533.7 \pm 24.8$ & $0.35 \pm 0.01$ & $=f_{9}+f_{22}$ \\
\hline$f_{27}$ & 0.7561 & 8.751 & $526.7 \pm 4.1$ & $0.36 \pm 0.00$ & $=f_{3}+f_{19},=f_{7}+f_{8}, \ldots$ \\
\hline$f_{28}$ & 0.3802 & 4.401 & $524.5 \pm 3.9$ & $0.91 \pm 0.00$ & \\
\hline$f_{29}$ & 0.0721 & 0.835 & $496.0 \pm 4.2$ & $0.27 \pm 0.00$ & $=f_{5} * 3$ \\
\hline$f_{30}$ & 0.3627 & 4.198 & $484.5 \pm 4.0$ & $0.21 \pm 0.00$ & $=f_{20} * 4$ \\
\hline$f_{31}$ & 0.7976 & 9.231 & $463.7 \pm 4.0$ & $0.11 \pm 0.00$ & \\
\hline$f_{32}$ & 1.0751 & 12.443 & $461.0 \pm 4.1$ & $0.70 \pm 0.00$ & \\
\hline$f_{33}$ & 2.4517 & 28.377 & $460.7 \pm 4.0$ & $0.21 \pm 0.00$ & $=f_{6} * 2, \sim f_{6}+2 \Delta f$ \\
\hline$f_{34}$ & 0.5528 & 6.399 & $460.4 \pm 4.0$ & $0.53 \pm 0.00$ & $=f_{14}+f_{20}$ \\
\hline$f_{35}$ & 1.3723 & 15.883 & $454.8 \pm 4.1$ & $0.46 \pm 0.00$ & $=f_{6}+f_{7}, \ldots$ \\
\hline$f_{36}$ & 0.6228 & 7.208 & $433.9 \pm 31.3$ & $0.61 \pm 0.01$ & $=f_{25} * 2$ \\
\hline$f_{37}$ & 1.1254 & 13.025 & $433.8 \pm 4.1$ & $0.38 \pm 0.00$ & \\
\hline$f_{38}$ & 1.3570 & 15.706 & $431.7 \pm 5.6$ & $0.81 \pm 0.00$ & $=f_{15} * 2$ \\
\hline$f_{39}$ & 0.5135 & 5.943 & $429.6 \pm 4.3$ & $0.82 \pm 0.00$ & \\
\hline$f_{40}$ & 1.0205 & 11.811 & $428.6 \pm 3.9$ & $0.77 \pm 0.00$ & \\
\hline$f_{41}$ & 0.5944 & 6.880 & $423.3 \pm 10.6$ & $0.97 \pm 0.00$ & \\
\hline$f_{42}$ & 0.8719 & 10.091 & $417.4 \pm 4.0$ & $0.73 \pm 0.00$ & \\
\hline$f_{43}$ & 0.4305 & 4.982 & $413.9 \pm 4.0$ & $0.92 \pm 0.00$ & \\
\hline$f_{44}$ & 1.1822 & 13.683 & $411.4 \pm 4.0$ & $0.67 \pm 0.00$ & \\
\hline$f_{45}$ & 1.2980 & 15.023 & $409.1 \pm 4.0$ & $0.32 \pm 0.00$ & \\
\hline$f_{46}$ & 1.1931 & 13.809 & $406.8 \pm 4.1$ & $0.52 \pm 0.00$ & \\
\hline$f_{47}$ & 2.2289 & 25.797 & $398.9 \pm 3.9$ & $0.47 \pm 0.00$ & \\
\hline$f_{48}$ & 0.2076 & 2.403 & $398.2 \pm 4.2$ & $0.77 \pm 0.00$ & \\
\hline$f_{49}$ & 1.6673 & 19.297 & $397.2 \pm 4.1$ & $0.96 \pm 0.00$ & \\
\hline$f_{50}$ & 2.0431 & 23.647 & $396.2 \pm 3.9$ & $0.81 \pm 0.00$ & \\
\hline$f_{51}$ & 1.3963 & 16.161 & $394.8 \pm 4.0$ & $0.37 \pm 0.00$ & \\
\hline$f_{52}$ & 1.7241 & 19.955 & $393.3 \pm 3.9$ & $0.23 \pm 0.00$ & \\
\hline$f_{53}$ & 1.2084 & 13.986 & $390.5 \pm 4.3$ & $0.11 \pm 0.00$ & \\
\hline$f_{54}$ & 1.8858 & 21.826 & $388.2 \pm 4.2$ & $0.79 \pm 0.00$ & \\
\hline$f_{55}$ & 1.0948 & 12.671 & $385.8 \pm 4.0$ & $0.16 \pm 0.00$ & \\
\hline$f_{56}$ & 0.7211 & 8.346 & $385.8 \pm 4.0$ & $0.18 \pm 0.00$ & \\
\hline$f_{57}$ & 1.8159 & 21.017 & $384.8 \pm 4.0$ & $0.97 \pm 0.00$ & \\
\hline$f_{58}$ & 0.7298 & 8.447 & $383.4 \pm 4.0$ & $0.43 \pm 0.00$ & \\
\hline$f_{59}$ & 0.3256 & 3.768 & $380.4 \pm 4.0$ & $0.46 \pm 0.00$ & \\
\hline$f_{60}$ & 0.9440 & 10.926 & $376.9 \pm 4.0$ & $0.14 \pm 0.00$ & \\
\hline$f_{61}$ & 0.2841 & 3.288 & $375.0 \pm 4.0$ & $0.40 \pm 0.00$ & \\
\hline
\end{tabular}

Notes. The minimum detectable frequency is $0.0087 \mathrm{c} \mathrm{d}^{-1}$, i.e., $0.1 \mu \mathrm{Hz}$. The error bar on the frequencies is $0.0022 \mathrm{c} \mathrm{d}{ }^{-1}$, i.e., $0.025 \mu \mathrm{Hz}$. The amplitudes and phases calculated with AмPHI are reported in Cols. 4 and 5 together with their error bars. The last column provides remarks about some frequencies. 
Table 3. continued.

\begin{tabular}{|c|c|c|c|c|}
\hline & $\begin{array}{l}\text { Frequency } \\
\mathrm{c} \mathrm{d}^{-1}\end{array}$ & $\mu \mathrm{Hz}$ & $\begin{array}{c}\text { Amplitude } \\
\mu \mathrm{mag}\end{array}$ & $\begin{array}{c}\text { Phase } \\
{[0: 1]}\end{array}$ \\
\hline$f_{62}$ & 0.5419 & 6.272 & $374.3 \pm 4.0$ & $0.55 \pm 0.00$ \\
\hline$f_{63}$ & 1.9492 & 22.560 & $371.7 \pm 3.9$ & $0.83 \pm 0.00$ \\
\hline$f_{64}$ & 1.3220 & 15.301 & $366.7 \pm 4.1$ & $0.90 \pm 0.00$ \\
\hline$f_{65}$ & 0.4960 & 5.741 & $366.2 \pm 4.1$ & $0.87 \pm 0.00$ \\
\hline$f_{66}$ & 1.3133 & 15.200 & $365.3 \pm 4.0$ & $0.15 \pm 0.00$ \\
\hline$f_{67}$ & 1.4225 & 16.465 & $352.7 \pm 3.9$ & $0.02 \pm 0.00$ \\
\hline$f_{68}$ & 0.7430 & 8.599 & $349.7 \pm 4.1$ & $0.76 \pm 0.00$ \\
\hline$f_{69}$ & 1.7984 & 20.815 & $346.5 \pm 4.0$ & $0.74 \pm 0.00$ \\
\hline$f_{70}$ & 1.5274 & 17.679 & $346.2 \pm 3.9$ & $0.78 \pm 0.00$ \\
\hline$f_{71}$ & 1.0292 & 11.912 & $343.6 \pm 3.9$ & $0.10 \pm 0.00$ \\
\hline$f_{72}$ & 0.4086 & 4.729 & $343.5 \pm 4.0$ & $0.92 \pm 0.00$ \\
\hline$f_{73}$ & 0.9921 & 11.482 & $341.6 \pm 4.0$ & $0.06 \pm 0.00$ \\
\hline$f_{74}$ & 0.1071 & 1.239 & $335.2 \pm 4.0$ & $0.19 \pm 0.00$ \\
\hline$f_{75}$ & 1.6498 & 19.095 & $332.4 \pm 4.0$ & $0.08 \pm 0.00$ \\
\hline$f_{76}$ & 0.9571 & 11.078 & $331.3 \pm 4.1$ & $0.78 \pm 0.00$ \\
\hline$f_{77}$ & 0.8850 & 10.243 & $330.4 \pm 4.0$ & $0.01 \pm 0.00$ \\
\hline$f_{78}$ & 1.4575 & 16.869 & $327.7 \pm 4.2$ & $0.16 \pm 0.00$ \\
\hline$f_{79}$ & 0.9047 & 10.471 & $316.0 \pm 3.9$ & $0.40 \pm 0.00$ \\
\hline$f_{80}$ & 1.5973 & 18.488 & $315.0 \pm 3.9$ & $0.79 \pm 0.00$ \\
\hline$f_{81}$ & 3.1073 & 35.964 & $312.0 \pm 4.0$ & $0.68 \pm 0.00$ \\
\hline$f_{82}$ & 1.6258 & 18.817 & $311.8 \pm 4.0$ & $0.17 \pm 0.00$ \\
\hline$f_{83}$ & 2.4212 & 28.023 & $311.0 \pm 3.9$ & $0.00 \pm 0.00$ \\
\hline$f_{84}$ & 0.3518 & 4.072 & $310.4 \pm 4.0$ & $0.08 \pm 0.00$ \\
\hline$f_{85}$ & 2.3425 & 27.112 & $309.4 \pm 3.9$ & $0.94 \pm 0.00$ \\
\hline$f_{86}$ & 0.1726 & 1.998 & $305.1 \pm 4.1$ & $0.73 \pm 0.00$ \\
\hline$f_{87}$ & 1.8923 & 21.902 & $298.6 \pm 4.2$ & $0.12 \pm 0.00$ \\
\hline$f_{88}$ & 0.0590 & 0.683 & $298.5 \pm 4.4$ & $0.42 \pm 0.00$ \\
\hline$f_{89}$ & 1.1472 & 13.278 & $297.8 \pm 4.0$ & $0.02 \pm 0.00$ \\
\hline$f_{90}$ & 3.1816 & 36.824 & $297.3 \pm 3.9$ & $0.15 \pm 0.00$ \\
\hline$f_{91}$ & 1.3351 & 15.453 & $295.7 \pm 4.1$ & $0.67 \pm 0.00$ \\
\hline$f_{92}$ & 0.5244 & 6.070 & $294.9 \pm 4.0$ & $0.79 \pm 0.00$ \\
\hline$f_{93}$ & 2.3337 & 27.012 & $294.0 \pm 3.9$ & $0.71 \pm 0.00$ \\
\hline$f_{94}$ & 0.3715 & 4.299 & $292.8 \pm 3.9$ & $0.21 \pm 0.00$ \\
\hline$f_{95}$ & 1.4859 & 17.198 & $289.8 \pm 4.2$ & $0.83 \pm 0.00$ \\
\hline$f_{96}$ & 0.1311 & 1.517 & $283.5 \pm 4.1$ & $0.59 \pm 0.00$ \\
\hline$f_{97}$ & 1.2892 & 14.922 & $282.4 \pm 4.1$ & $0.57 \pm 0.00$ \\
\hline$f_{98}$ & 0.5878 & 6.803 & $279.0 \pm 7.4$ & $0.36 \pm 0.00$ \\
\hline$f_{99}$ & 2.2048 & 25.519 & $273.9 \pm 3.9$ & $0.59 \pm 0.00$ \\
\hline$f_{100}$ & 1.2018 & 13.910 & $273.9 \pm 4.3$ & $0.72 \pm 0.00$ \\
\hline$f_{101}$ & 0.2273 & 2.630 & $270.3 \pm 3.9$ & $0.22 \pm 0.00$ \\
\hline$f_{102}$ & 1.1363 & 13.151 & $269.1 \pm 4.0$ & $0.19 \pm 0.00$ \\
\hline$f_{103}$ & 0.5070 & 5.868 & $267.8 \pm 4.2$ & $0.88 \pm 0.00$ \\
\hline$f_{104}$ & 0.7714 & 8.928 & $262.6 \pm 4.0$ & $0.38 \pm 0.00$ \\
\hline$f_{105}$ & 1.6848 & 19.500 & $260.7 \pm 4.0$ & $0.08 \pm 0.00$ \\
\hline$f_{106}$ & 2.5697 & 29.742 & $260.3 \pm 4.0$ & $0.20 \pm 0.00$ \\
\hline$f_{107}$ & 1.9076 & 22.079 & $260.2 \pm 4.0$ & $0.90 \pm 0.00$ \\
\hline$f_{108}$ & 3.1204 & 36.116 & $259.7 \pm 4.0$ & $0.05 \pm 0.00$ \\
\hline$f_{109}$ & 1.0532 & 12.190 & $257.5 \pm 4.0$ & $0.86 \pm 0.00$ \\
\hline$f_{110}$ & 1.3810 & 15.984 & $254.3 \pm 4.0$ & $0.28 \pm 0.00$ \\
\hline$f_{111}$ & 1.8355 & 21.245 & $252.4 \pm 4.3$ & $0.02 \pm 0.00$ \\
\hline$f_{112}$ & 1.8508 & 21.422 & $250.2 \pm 4.0$ & $0.71 \pm 0.00$ \\
\hline$f_{113}$ & 1.9251 & 22.282 & $248.8 \pm 3.9$ & $0.42 \pm 0.00$ \\
\hline$f_{114}$ & 1.8683 & 21.624 & $247.6 \pm 3.9$ & $0.48 \pm 0.00$ \\
\hline$f_{115}$ & 0.2578 & 2.984 & $244.0 \pm 4.0$ & $0.55 \pm 0.00$ \\
\hline$f_{116}$ & 2.8254 & 32.701 & $243.1 \pm 3.9$ & $0.26 \pm 0.00$ \\
\hline$f_{117}$ & 2.6724 & 30.931 & $242.1 \pm 3.9$ & $0.69 \pm 0.00$ \\
\hline$f_{118}$ & 0.5725 & 6.626 & $241.5 \pm 4.2$ & $0.67 \pm 0.00$ \\
\hline$f_{119}$ & 1.4509 & 16.793 & $240.4 \pm 4.2$ & $0.24 \pm 0.00$ \\
\hline$f_{120}$ & 1.9579 & 22.661 & $239.8 \pm 3.9$ & $0.11 \pm 0.00$ \\
\hline$f_{121}$ & 2.4649 & 28.528 & $238.7 \pm 4.0$ & $0.93 \pm 0.00$ \\
\hline$f_{122}$ & 3.5705 & 41.326 & $238.4 \pm 3.9$ & $0.22 \pm 0.00$ \\
\hline$f_{123}$ & 1.7001 & 19.677 & $238.1 \pm 4.0$ & $0.43 \pm 0.00$ \\
\hline$f_{124}$ & 0.1836 & 2.124 & $237.4 \pm 4.0$ & $0.88 \pm 0.00$ \\
\hline$f_{125}$ & 2.0584 & 23.824 & $235.2 \pm 4.1$ & $0.90 \pm 0.00$ \\
\hline$f_{126}$ & 0.9702 & 11.229 & $235.1 \pm 4.1$ & $0.28 \pm 0.00$ \\
\hline$f_{127}$ & 1.5012 & 17.375 & $233.9 \pm 4.0$ & $0.52 \pm 0.00$ \\
\hline
\end{tabular}


Table 3. continued.

\begin{tabular}{|c|c|c|c|c|}
\hline & $\begin{array}{l}\text { Frequency } \\
\mathrm{c} \mathrm{d}^{-1}\end{array}$ & $\mu \mathrm{Hz}$ & $\begin{array}{c}\text { Amplitude } \\
\mu \mathrm{mag}\end{array}$ & $\begin{array}{c}\text { Phase } \\
{[0: 1]}\end{array}$ \\
\hline$f_{128}$ & 2.7293 & 31.589 & $233.2 \pm 3.9$ & $0.23 \pm 0.00$ \\
\hline$f_{129}$ & 0.3890 & 4.502 & $232.8 \pm 3.9$ & $0.28 \pm 0.00$ \\
\hline$f_{130}$ & 2.8516 & 33.005 & $232.7 \pm 3.9$ & $0.69 \pm 0.00$ \\
\hline$f_{131}$ & 0.8216 & 9.509 & $228.9 \pm 4.0$ & $0.57 \pm 0.00$ \\
\hline$f_{132}$ & 0.4807 & 5.564 & $227.1 \pm 4.0$ & $0.08 \pm 0.00$ \\
\hline$f_{133}$ & 2.5829 & 29.894 & $226.8 \pm 4.0$ & $0.74 \pm 0.00$ \\
\hline$f_{134}$ & 2.3665 & 27.390 & $226.5 \pm 3.9$ & $0.50 \pm 0.00$ \\
\hline$f_{135}$ & 1.6345 & 18.918 & $222.9 \pm 4.0$ & $0.01 \pm 0.00$ \\
\hline$f_{136}$ & 0.2010 & 2.327 & $222.8 \pm 4.1$ & $0.94 \pm 0.00$ \\
\hline$f_{137}$ & 3.3979 & 39.328 & $222.7 \pm 3.9$ & $0.09 \pm 0.00$ \\
\hline$f_{138}$ & 1.6738 & 19.373 & $222.1 \pm 4.2$ & $0.89 \pm 0.00$ \\
\hline$f_{139}$ & 0.2447 & 2.833 & $221.2 \pm 4.0$ & $0.23 \pm 0.00$ \\
\hline$f_{140}$ & 1.1122 & 12.873 & $220.9 \pm 4.3$ & $0.80 \pm 0.00$ \\
\hline$f_{141}$ & 0.2950 & 3.414 & $220.8 \pm 4.1$ & $0.39 \pm 0.00$ \\
\hline$f_{142}$ & 3.0417 & 35.205 & $217.6 \pm 3.9$ & $0.69 \pm 0.00$ \\
\hline$f_{143}$ & 2.0519 & 23.748 & $215.0 \pm 4.1$ & $0.01 \pm 0.00$ \\
\hline$f_{144}$ & 0.0393 & 0.455 & $213.9 \pm 4.1$ & $0.69 \pm 0.00$ \\
\hline$f_{145}$ & 1.2390 & 14.340 & $212.2 \pm 4.9$ & $0.72 \pm 0.00$ \\
\hline$f_{146}$ & 0.9811 & 11.356 & $211.4 \pm 4.0$ & $0.27 \pm 0.00$ \\
\hline$f_{147}$ & 0.1442 & 1.669 & $211.3 \pm 9.4$ & $0.06 \pm 0.01$ \\
\hline$f_{148}$ & 3.6186 & 41.882 & $211.3 \pm 3.9$ & $0.19 \pm 0.00$ \\
\hline$f_{149}$ & 1.0117 & 11.710 & $211.1 \pm 4.0$ & $0.29 \pm 0.00$ \\
\hline$f_{150}$ & 0.9134 & 10.572 & $208.7 \pm 3.9$ & $0.68 \pm 0.00$ \\
\hline$f_{151}$ & 2.6921 & 31.159 & $208.4 \pm 3.9$ & $0.16 \pm 0.00$ \\
\hline$f_{152}$ & 2.0956 & 24.254 & $205.0 \pm 3.9$ & $0.53 \pm 0.00$ \\
\hline$f_{153}$ & 2.1415 & 24.785 & $204.9 \pm 3.9$ & $0.28 \pm 0.00$ \\
\hline$f_{154}$ & 0.7867 & 9.105 & $204.7 \pm 4.0$ & $0.99 \pm 0.00$ \\
\hline$f_{155}$ & 2.2529 & 26.075 & $204.7 \pm 3.9$ & $0.69 \pm 0.00$ \\
\hline$f_{156}$ & 1.4400 & 16.667 & $204.0 \pm 4.0$ & $0.20 \pm 0.00$ \\
\hline$f_{157}$ & 1.1560 & 13.379 & $203.2 \pm 3.9$ & $0.17 \pm 0.00$ \\
\hline$f_{158}$ & 4.5648 & 52.833 & $201.0 \pm 3.9$ & $0.35 \pm 0.00$ \\
\hline$f_{159}$ & 2.5086 & 29.034 & $201.0 \pm 3.9$ & $0.90 \pm 0.00$ \\
\hline$f_{160}$ & 1.5624 & 18.083 & $199.9 \pm 3.9$ & $0.76 \pm 0.00$ \\
\hline$f_{161}$ & 2.4867 & 28.781 & $199.3 \pm 3.9$ & $0.37 \pm 0.00$ \\
\hline$f_{162}$ & 2.8691 & 33.207 & $198.6 \pm 3.9$ & $0.08 \pm 0.00$ \\
\hline$f_{163}$ & 3.7344 & 43.223 & $197.6 \pm 3.9$ & $0.27 \pm 0.00$ \\
\hline$f_{164}$ & 1.2761 & 14.770 & $196.5 \pm 4.1$ & $0.56 \pm 0.00$ \\
\hline$f_{165}$ & 3.6579 & 42.337 & $192.8 \pm 3.9$ & $0.86 \pm 0.00$ \\
\hline$f_{166}$ & 2.7533 & 31.867 & $191.8 \pm 3.9$ & $0.85 \pm 0.00$ \\
\hline$f_{167}$ & 3.6973 & 42.793 & $191.8 \pm 3.9$ & $0.13 \pm 0.00$ \\
\hline$f_{168}$ & 0.0852 & 0.986 & $191.6 \pm 5.5$ & $0.87 \pm 0.00$ \\
\hline$f_{169}$ & 2.7839 & 32.221 & $191.6 \pm 3.9$ & $0.91 \pm 0.00$ \\
\hline$f_{170}$ & 1.4793 & 17.122 & $190.6 \pm 4.3$ & $0.99 \pm 0.00$ \\
\hline$f_{171}$ & 2.2201 & 25.696 & $189.2 \pm 3.9$ & $0.57 \pm 0.00$ \\
\hline$f_{172}$ & 1.3526 & 15.655 & $188.5 \pm 5.5$ & $0.19 \pm 0.00$ \\
\hline$f_{173}$ & 2.5391 & 29.388 & $187.8 \pm 3.9$ & $0.77 \pm 0.00$ \\
\hline$f_{174}$ & 3.3608 & 38.898 & $187.6 \pm 3.9$ & $0.76 \pm 0.00$ \\
\hline$f_{175}$ & 2.9915 & 34.624 & $187.6 \pm 3.9$ & $0.50 \pm 0.00$ \\
\hline$f_{176}$ & 2.2682 & 26.252 & $186.5 \pm 3.9$ & $0.40 \pm 0.00$ \\
\hline$f_{177}$ & 1.6148 & 18.690 & $185.9 \pm 4.0$ & $0.27 \pm 0.00$ \\
\hline$f_{178}$ & 1.7503 & 20.258 & $185.8 \pm 3.9$ & $0.23 \pm 0.00$ \\
\hline$f_{179}$ & 4.4818 & 51.872 & $185.6 \pm 3.9$ & $0.68 \pm 0.00$ \\
\hline$f_{180}$ & 3.6033 & 41.705 & $183.4 \pm 3.9$ & $0.79 \pm 0.00$ \\
\hline$f_{181}$ & 0.3168 & 3.667 & $183.0 \pm 4.2$ & $0.28 \pm 0.00$ \\
\hline$f_{182}$ & 0.8063 & 9.332 & $182.1 \pm 4.0$ & $0.76 \pm 0.00$ \\
\hline$f_{183}$ & 4.2436 & 49.115 & $178.6 \pm 3.9$ & $0.37 \pm 0.00$ \\
\hline$f_{184}$ & 2.9303 & 33.915 & $176.9 \pm 3.9$ & $0.84 \pm 0.00$ \\
\hline$f_{185}$ & 3.0002 & 34.725 & $176.3 \pm 3.9$ & $0.69 \pm 0.00$ \\
\hline$f_{186}$ & 1.7875 & 20.688 & $174.3 \pm 4.0$ & $0.81 \pm 0.00$ \\
\hline$f_{187}$ & 2.3906 & 27.669 & $173.8 \pm 3.9$ & $0.35 \pm 0.00$ \\
\hline$f_{188}$ & 0.4698 & 5.438 & $153.5 \pm 5.3$ & $0.55 \pm 0.01$ \\
\hline$f_{189}$ & 1.2281 & 14.214 & $62.8 \pm 12.5$ & $0.80 \pm 0.03$ \\
\hline
\end{tabular}

